\title{
Study of Fuzzified Boundary Value Problems for MHD Couette and Poiseuille Flow of a Differential Type Fluid
}

\section{Muhammad Nadeem}

The University of Lahore

Imran Siddique

The University of Lahore

Raja Noshad Jamil

The University of Lahore

\section{Ali Akgül ( $\square$ aliakgul00727@gmail.com )}

Siirt University: Siirt Universitesi https://orcid.org/0000-0001-9832-1424

\section{Research Article}

Keywords: Triangular fuzzy numbers (TFNs), differential type fluid, Adomian decomposition method (ADM), Couette flow, Poiseuille flow.

Posted Date: June 16th, 2021

DOl: https://doi.org/10.21203/rs.3.rs-172672/v1

License: (9) This work is licensed under a Creative Commons Attribution 4.0 International License. Read Full License 


\title{
Study of Fuzzified Boundary Value Problems for MHD Couette and Poiseuille Flow of a Differential Type Fluid
}

\author{
${ }^{1}$ Muhammad Nadeem, ${ }^{1}$ Imran Siddique, ${ }^{1}$ Raja Noshad Jamil and ${ }^{2 *}$ Ali Akgül \\ ${ }^{1}$ Department of Mathematics, University of Management and Technology, Lahore, Pakistan \\ ${ }^{2}$ Department of Mathematics, Art and Science Faculty, Siirt University, TR-56100 Siirt, Turkey
}

(*): aliakgu100727@gmail.com

\begin{abstract}
In this work, discuss the magneto-hydro-dynamics (MHD) flow in three fundamental flaws of the third-grade fluid between two parallel plates in a fuzzy environment by the fuzzy Adomian decomposition method (ADM). We extend the work of Kamran and Siddique [19], using fuzzy differential equations (FDEs) and explain our approach with the help of membership function of triangular fuzzy numbers (TFNs). In the end, the effect of the fuzzy parameter $(\alpha \in[0,1])$, and other engineering parameters on fuzzy velocity profiles are investigating in graphically and tabular representation.
\end{abstract}

Keywords: Triangular fuzzy numbers (TFNs); differential type fluid; Adomian decomposition method (ADM); Couette flow; Poiseuille flow.

AMS Mathematics Subject Classification: 76A05, 76Mxx.

\section{INTRODUCTION}

The study of non-Newtonian fluids has gained considerable attention to scientists because of extensive applications in engineering, science and industry. Various industrial ingredients fall into this bunch, such as biological solutions, soap, paints, cosmetics, tars, shampoos, mayonnaise, blood, yogurt, syrups, and glues, etc. Due to the intricate nature of non-Newtonian fluid, it's very hard to establish a single model that can describe characteristics of all nonNewtonian fluid. So, the fluids of differential type [1] have received superior consideration by researches. Here, we will consider the third-grade fluids (differential type by a subclass), which have been studied effectively in numerous types of flow mechanisms [2-9]. In fluid dynamics, 
the study of three fundamental flows (namely, Couette, Poiseuille, and generalized Couette flow) attracts the researchers by various non-Newtonian fluids, because of their uses in engineering and industry. The unidirectional flows are used in polymer engineering for instance die flow, injection molding, extrusion, plastic forming, continuous casting, and asthenosphere flows [1013]. Magneto-hydrodynamics (MHD) deals with the study of the motion of electrically conducting fluids in the presence of the magnetic field. MHD flow has significant importance applications between infinite parallel plates in various areas such as geophysical, astrophysical, metallurgical processing, MHD generators, pumps, geothermal reservoirs, polymer technology, and mineral industries, etc. MHD fluids use as a lubricant in industrial and other applications, for stops the unexpected variation of lubricant viscosity with temperature under the certain norms. In this way there is large number of literature as Khan et al. [14], Hayat et al. [15, 16, and 17], and Islam et al. [18]. Kamran and Siddique [19] calculated the analytical solutions for MHD flow between infinite parallel plates.

Adomian [20-22] introduced the method namely Adomian Decomposition Method (ADM). $\mathrm{ADM}$ is reliable and effective technique to compute the linear and non-linear differential equations. So, it gives analytical solutions in the form of an infinite convergent series. The ADM has some vital advantages over other analytical methods as well as numerical methods, needs no linearization, discretization, perturbation, and spatial transformation. Siddiqui et al., [23] deliberated parallel plate flow of a third grade fluid using ADM and compare the results with numerical technique. Pirzada and Vakaskar [24] calculated the solution of the fuzzy heat equation with the help of fuzzy ADM. Paripour et al., [25] studied the analytical solution of hybrid FDEs by using the fuzzy ADM and predictor-corrector method, which shows ADM is better than the predictor-corrector method. Also, Siddiquie et al., [26] provided a comparison of ADM and homotopy perturbation method (HPM) in terms of squeezing flow between two circular plates. Their analysis shows the ADM is better than HPM. Biswal et al. [27] studied Natural convection of nanofluid flow between two parallel plates using HPM in a fuzzy environment. The volume fraction of nanoparticle considered as TFN and also shows the fuzzy result is better than a crisp result.

Fluid flow plays a main role in the field of science and engineering. The rise in an extensive range of problems like chemical diffusion, magnetic effect and heat transfer etc. After governing these physical problems convert into linear or nonlinear DEs. In general, the physical problems with 
involved geometry, coefficients, parameters, initial, and boundary conditions greatly affect the solution of DEs. Then the coefficients, parameters, initial, and boundary conditions are not crisp due to the mechanical defect, experimental error, and measurement error etc. So in this situation, fuzzy sets theory is an effective tool for a better understanding of the considered phenomena and it is more accurate than assuming the crisp or classical physical problems. For more precisely the FDEs play a major role to reduce the uncertainty and proper way to describe the physical problem which arise in uncertain parameters, initial and boundary conditions.

In 1965, Zadeh [28] presented the fuzzy set theory (FST). FST is very valuable tool to define the situation in which information is imprecise, vague or uncertain. FST is completely defined by its membership function or belongingness. In FST, the membership function describes each element of the universe of discourse by a number from $[0,1]$ interval. On the other hand, the degree of nonbelongingness is a complement to "one" of the membership degree or belongingness. Fuzzy number $(\mathrm{FN})$ can be expected as a function whose range is specified zero to one. Every numerical value in the range is allocated a definite grade of membership function where, 0 signifies the minimum possible grade and 1 is the maximum possible grade. As FST is the generalization of crisp (classical) set theory and similarly, FNs are the generalization of real intervals. Arithmetic operations on FNs are developed by Dubois and Prade [29]. Different types of FNs can be categories in triangular, trapezoidal and Gaussian fuzzy numbers. Here we consider TFNs for the sake of completeness.

The modeling of physical problems in terms of ordinary differential equations (ODEs) includes uncertain parameters or variables. This impreciseness or vagueness can be defined mathematically using FNs. Seikala [30] introduced the fuzzy differentiability concept. Later on, Kaleva [31] presented the fuzzy differentiation and integration. Kandel and Byatt [32] introduced the FDEs in 1987. Buckleyet et al., [33] used two methods extension principle and FNs for the solution of FDEs. Nieto [34] studied the Cauchy problem for continuous FDEs. Lakshmikantham and Mohapatra studied the initial value problems for FDEs has been commenced in [35]. Salahsour et al. [36] studied the fuzzy logistic equation and alley effect using FDE with the help of TFNs. Hashemi et al. [37] used HAM to calculate the analytical solutions for system of fuzzy differential equations (SFDEs). Tzimopoulos et al. [38] studied the solution of vertical infiltration equation using FDE and get well-known results in a fuzzy environment. Gasilov et al., [39] developed the geometric method to solve SFDEs. Khastan and Nieto [40] used 
generalized differentiability concept to solve the second order FDE. In few decades ago, there have been many studies revolving around the concept of "fuzzy differential equations". Many scholars have applied FST to obtained well-known results in the field of commerce and science. Such as, bank account model [41], HIV model [42], Bacteria culture model [43], population dynamics model [44, 45], predator-prey model [46], computational biology [47], Decay model [48], modeling of hydraulic [49], Friction model [50], quantum optics and gravity [51], application in Laplace transform [52], civil engineering [53], Integro-differential equation [54], Giving up smoking model [55], Chemo-stat model [56], Dengue virus model [57]. Fuzzy IRD-14 days COVID-19 model [58]. Biswal et al. [59] studied Natural convection of nanofluid flow between two parallel plates using HPM in uncertain environment. The volume fraction of nanoparticle considered as TFN and also shows the fuzzy result is better than a crisp result.

In the review of literature, third-grade problems studied for only crisp or classical cases. So, the above-mentioned works motivated us to develop a model to describe the fuzzy analysis for unidirectional MHD flow in three fundamental flow problems namely, plane Couette, fully developed plane Poiseuille and plane Couette-Poiseuille flow of a third-grade fluid between two parallel plates. The basic purpose of this article is to show the uncertain flow mechanism through FDEs and generalize the work of Kamran and Siddique [19] in the circumstance of fuzzy environment.

The article is structured as follows; basic preliminaries are given in section 2. In section 3, developed the governing equations of the proposed study and changed governing equations in the fuzzy form for solving by a fuzzy ADM. Results and discussion in graphical with tabular forms are presented in section 4. In section 5, some conclusions are given.

\section{Preliminaries}

2.1 Definition [27]: Fuzzy set $\bar{Z}$ is defined as set of ordered pairs such that $\bar{Z}=\left\{\left(x, \mu_{\bar{Z}}(x)\right): x \in U, \mu_{\bar{Z}}(x) \in[0,1]\right\}$, here $U$ is the universal set, $\mu_{\bar{Z}}(x)$ is membership function of $\bar{Z}$ and mapping defined as $\mu_{\bar{Z}}(x): U \rightarrow[0,1]$.

2.2 Definition [28]: $\alpha$-cut or $\alpha$-level of a fuzzy set $\bar{Z}$, defined by $Z_{\alpha}=\left\{x / \mu_{\bar{Z}}(x) \geq \alpha\right\}$, where $Z_{\alpha}$ is crisp set and $0 \leq \alpha \leq 1$. 
2.3 Definition [27]: Convex fuzzy set $\bar{Z}$ is defined as $\bar{Z}=\left\{x, \mu_{\bar{Z}}(x)\right\} \subseteq U$ and all $Z_{\alpha}$ for every $0 \leq \alpha \leq 1$ are convex fuzzy set i.e. for every $y_{1}, y_{2} \in Z_{\alpha}$ and $\alpha y_{1}+(1-\alpha) y_{2} \in Z_{\alpha}$. Otherwise nonconvex fuzzy set.

2.4 Definition [27]: Fuzzy number $\bar{Z}$ defined as $\bar{Z}: R \rightarrow[0,1]$, is called a FN, satisfies the subsequent properties:

(i) $\mu_{\bar{Z}}(x)$ is piecewise continuous.

(ii) $\bar{Z}$ is convex.

(iii) $\bar{Z}$ is normal i.e. so their exist $x_{0} \in R$ such that $\mu_{\bar{Z}}\left(x_{0}\right)=1$.

(iv) Support of $\bar{Z}$ must be bounded.

(v) $Z_{\alpha}$ must be closed interval for every $0 \leq \alpha \leq 1$ also $\alpha$, is called level of credibility or presumption. Membership function or grade is also named as grade of possibility or grade of credibility for a given number.

2.5 Definition [28]: Let $\bar{Z}=(\delta, \chi, \eta)$ with membership function $\mu_{\bar{Z}}(x)$ is called a TFNs if

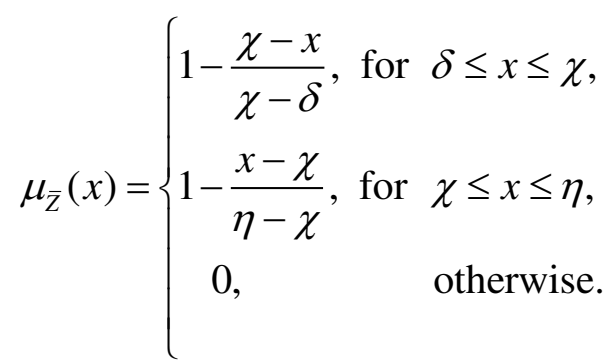

The TFNs with peak (center) $\chi$, right width $\eta-\chi>0$, left width $\chi-\delta>0$, and these TFNs are transformed into interval numbers through $\alpha$-cut approach, is written as $\bar{Z}=[v(x ; \alpha), u(x ; \alpha)]=[\delta+\alpha(\chi-\delta), \eta-\alpha(\eta-\chi)]$, where $0 \leq \alpha \leq 1$. TFNs satisfy the following conditions:

(i) $v(x ; \alpha)$ is non- decreasing on $[0,1]$.

(ii) $u(x ; \alpha)$ is non-increasing on $[0,1]$.

(iii) $u(x ; \alpha) \geq v(x ; \alpha)$ on $[0,1]$.

(iv) $v(x ; \alpha)$ and $u(x ; \alpha)$ are bounded on left continuous and right continuous at $[0,1]$ respectively. 
2.6 Definition [29]: Let $I^{\mathrm{m}}$ be an interval and $I^{\mathrm{m}} \subseteq R$. A mapping $\bar{u}: I^{\mathrm{m}} \rightarrow F^{*}$ is called a fuzzy process, defined as $\bar{u}(x ; \alpha)=[v(x ; \alpha), u(x ; \alpha)], x \in I^{\mathrm{m}}$ and $0 \leq \alpha \leq 1$. The derivative $\frac{d \bar{u}(x ; \alpha)}{d x} \in F^{*}$ of a fuzzy process $\bar{u}(x ; \alpha)$ is defined as $\frac{d \bar{u}(x ; \alpha)}{d x}=\left[\frac{d v(x ; \alpha)}{d x}, \frac{d u(x ; \alpha)}{d x}\right]$.

2.7 Definition [29]: Let $I^{\mathrm{m}} \subseteq R$ and $\bar{u}(x)$ be a fuzzy valued function define on $I^{\mathrm{m}}$. Let $\bar{u}(x ; \alpha)=[v(x ; \alpha), u(x ; \alpha)]$ for all $\alpha$-cut. Assume that $v(x ; \alpha)$ and $u(x ; \alpha)$ have continuous derivatives or differentiable, for all $x \in I^{\mathrm{m}}$ and $0 \leq \alpha \leq 1$ then $\left[\frac{d \bar{u}(x ; \alpha)}{d x}\right]_{\alpha}=\left[\frac{d v(x ; \alpha)}{d x}, \frac{d u(x ; \alpha)}{d x}\right]_{\alpha}$. Similarly, we can define higher order derivatives in same way. Then $\left[\frac{d \bar{u}(x ; \alpha)}{d x}\right]$, satisfy the following conditions:

(i) $\frac{d u(x ; \alpha)}{d x}$ and $\frac{d v(x ; \alpha)}{d x}$ are continuous on $[0,1]$.

(ii) $\frac{d v(x ; \alpha)}{d x}$ is non- decreasing on $[0,1]$.

(iii) $\frac{d u(x ; \alpha)}{d x}$ is non-increasing on $[0,1]$.

(iv) $\frac{d u(x ; \alpha)}{d x} \geq \frac{d v(x ; \alpha)}{d x}$ on $[0,1]$.

\section{Basic equations and formulation of the problem}

Let us consider the steady flow of a third-grade fluid between two horizontal parallel plates at distance $d$ apart. A uniform magnetic field is applied transversely to the plates. We choose coordinate system in which the $\mathrm{x}$-axis is taken perpendicular and $\mathrm{y}$-axis is parallel to the plates. For incompressible fluid the basic equations are:

$\operatorname{div} \mathbf{V}=0$.

$\rho \frac{D \mathbf{V}}{D t}=\operatorname{div} \tau^{*}+\rho \mathbf{f}+\mathbf{J} \times \mathbf{B}$, 
where $\rho$ is constant density, $\mathbf{V}$ is the velocity vector, $\mathbf{f}$ represents the body force per unit mass, $\tau^{*}$ is the stress tensor, $\mathbf{J}$ is the electric current density, $\mu$ is the viscosity, $\mathbf{B}$ is the total magnetic field. $\mathbf{B}=\mathbf{B}_{\mathrm{o}}+\mathbf{b}, \mathbf{B}_{\mathrm{o}}$ denotes the imposed magnetic field and $\mathbf{b}$ represents the induced magnetic field and $D(*) / D t=\partial(*) / \partial t+(\mathbf{V} \cdot \operatorname{grad})(*)$ is the material time derivative. In the absence of displacement currents, the modified Ohm's law and Maxwell's equations [19] are

$$
\begin{aligned}
& \mathbf{J}=\sigma[\mathbf{V} \times \mathbf{B}+\mathbf{E}], \\
& \nabla \times \mathbf{B}=\mu_{m} \mathbf{J}, \quad \nabla \times \mathbf{E}=-\frac{\partial \mathbf{B}}{\partial t}, \quad \nabla \mathbf{g} \mathbf{B}=0,
\end{aligned}
$$

wherein, $\mathbf{E}$ is the electric field, $\mu_{m}$ is the magnetic permeability and $\sigma$ is the electrical conductivity.

From Ohm's law and Maxwell's equations an evolution for the magnetic flux $\mathbf{B}$ can be obtained easily. This is known as the magnetic induction equation and it suggests that the motion of an electrically conducting fluid in an applied magnetic field induces a magnetic field in the medium. We assume that the total magnetic field $\mathbf{B}$ is perpendicular to the velocity field $\mathbf{V}$ and the induced magnetic field $\mathbf{b}$ is negligible compared with the applied magnetic field $\mathbf{B}_{\mathrm{o}}$, so that magnetic Reynolds number is small. Since no external electric field is applied and the effect of polarization of the ionized fluid is negligible, the fluid flow region is assumed as no electric field. Under these assumptions, the MHD force involved in Eq. (2) can be put into the form [19]

$$
\mathbf{J} \times \mathbf{B}=-\sigma \mathbf{B}_{0}^{2} \mathbf{V}
$$

For third-grade fluid, the stress tensor $\tau^{*}$ is given by [1-3]

$$
\tau^{*}=-p \mathbf{I}+\mu \mathbf{A}_{1}+\alpha_{1} \mathbf{A}_{2}+\alpha_{2} \mathbf{A}_{1}^{2}+\beta_{1} \mathbf{A}_{3}+\beta_{2}\left(\mathbf{A}_{2} \mathbf{A}_{1}+\mathbf{A}_{1} \mathbf{A}_{2}\right)+\beta_{3}\left(\operatorname{tr} \mathbf{A}_{2}\right) \mathbf{A}_{1},
$$

where $\mu$ is the coefficient of viscosity, $p$ is the pressure, $\mathbf{I}$ is the unit tensor, $\alpha_{1}, \alpha_{2}, \beta_{1}, \beta_{2}$ and $\beta_{3}$ are material constants, $\mathbf{A}_{1}, \mathbf{A}_{2}$ and $\mathbf{A}_{3}$ are the Rivlin-Ericksen tensors given by 


$$
\begin{aligned}
& \mathbf{A}_{1}=(\operatorname{grad} \mathbf{V})+(\operatorname{grad} \mathbf{V})^{t}, \\
& \mathbf{A}_{n}=\frac{D \mathbf{A}_{n-1}}{D t}+\mathbf{A}_{n-1}(\operatorname{grad} \mathbf{V})+(\operatorname{grad} \mathbf{V})^{t} \mathbf{A}_{n-1}, n \geq 1 .
\end{aligned}
$$

The flow is one dimensional, we define velocity field in component form as:

$$
\mathbf{V}=(u(x), 0,0)
$$

By using the above assumptions and Eq. (7), continuity Eq. (1) is identically satisfied and the momentum Eq. (2) in the absence of body forces reduces to the forms

$$
\begin{aligned}
& -\frac{d p}{d y}+\mu \frac{d^{2} u}{d x^{2}}+6\left(\beta_{2}+\beta_{3}\right) \frac{d^{2} u}{d x^{2}}\left(\frac{d u}{d x}\right)^{2}-\sigma B_{0}^{2} u=0 \\
& -\frac{d p}{d x}+\left(2 \alpha_{1}+\alpha_{2}\right) \frac{d}{d x}\left(\left(\frac{d u}{d x}\right)^{2}\right)=0 .
\end{aligned}
$$

On introducing the modified pressure $p^{\mu}$

$$
p^{ \pm}=-p+\left(2 \alpha_{1}+\alpha_{2}\right)\left(\left(\frac{d u}{d x}\right)^{2}\right)
$$

into Eq. (9) we have

$$
\frac{d p^{ \pm}}{d x}=0
$$

From Eq. (11), $p^{ \pm}=p^{ \pm}(y)$ and consequently, Eq. (8) become

$$
-\frac{1}{\mu} \frac{d p^{ \pm}}{d y}+\frac{d^{2} u}{d x^{2}}+6 \frac{\left(\beta_{2}+\beta_{3}\right)}{\mu}\left(\frac{d u}{d x}\right)^{2} \frac{d^{2} u}{d x^{2}}-\frac{\sigma B_{0}^{2} u}{\mu}=0 .
$$

Eq. (12) is a second-order non-linear ordinary differential equation.

\section{Adomian decomposition method}

In this section, we discuss the basic sketch of ADM and write the basic non-linear differential equation as 
$L_{1} u^{ \pm}(x)+N_{1} u^{ \pm}(x)=q(x)$

where $q, L_{1}$ and $N_{1}$ are source term, linear and non-linear operators respectively. Also the operator $L_{1}$ can be written as

$L_{1}=R_{1}+\hat{L}$

here, $\hat{L}$ is the highest order derivative in $L_{1}$ and is assumed to be easily invertible, $R_{1}$ is the remaining operator in $L_{1}$ whose order is less than the order of $\hat{L}$. From Eqs. (13) and (14) we have

$\hat{L} u^{ \pm}(x)=q(x)-R_{1} u^{ \pm}(x)-N_{1} u^{ \pm}(x)$.

Applying $\hat{L}^{-1}$ on the above Eq. (14), we have

$u^{ \pm}(x)=-\hat{L}^{-1} R_{1} u^{ \pm}(x)-\hat{L}^{-1} N_{1} u^{ \pm}(x)+g(x)$,

where $g(x)$ signifies the terms arising after integration of $q(x)$ and calculate constants of integration with the help of boundary conditions. So, $u^{ \pm}(x)$ and $N_{1} u^{ \pm}(x)$ can be write as [2022],

$u^{ \pm}(x)=\sum_{n=0}^{\infty} u_{n}^{ \pm}(x)$

$N_{1} u(x)=\sum_{n=0}^{\infty} A_{n}^{*}(x)$

where $A_{n, s}^{*}$ are called Adomian polynomials [20-21].

The algorithm of the general ADM can be communicated as

$u_{0}^{ \pm}(x)=g(x)$

$u_{n+1}{ }^{ \pm}(x)=-\hat{L}^{-1} A_{n}^{*}(x)-\hat{L}^{-1} R_{1} u_{n}^{ \pm}(x), \quad n \geq 0$.

$u^{ \pm}(x)=\sum_{k=1}^{\infty} u_{k}^{ \pm}(x)$

Many researchers have been established the convergence of this method [22]. In this continuation, we apply the ADM in fuzzy sense to three flow problems.

\subsection{Plane Couette flow}


Let us consider the steady flow of an incompressible third grade fluid between two horizontal infinite parallel plates. The lower plate is fixed while, the upper plate at $x=d$ is moving with uniform velocity $U$. The plates are non-conducting and a magnetic field is applied in the vertical upward direction. Let $\mathrm{y}$-axis be taken normal direction to flow and $\mathrm{x}$-axis in the direction of flow (See in Fig. 1). In the absence of pressure gradient, the differential equation (12) for such a flow reduces to

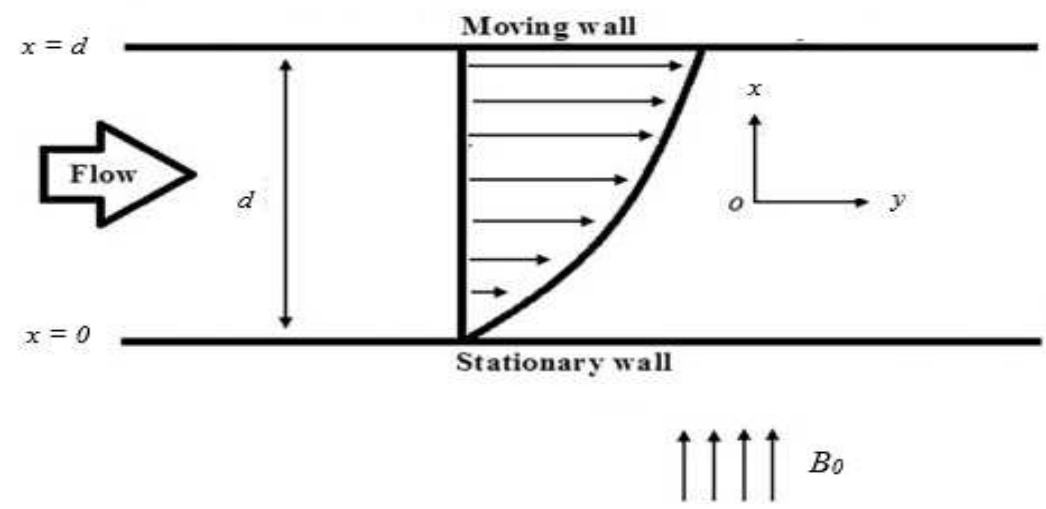

Fig. 1 Geometry of the couette and generalized couette flow.

$\frac{d^{2} u}{d x^{2}}+6 \frac{\left(\beta_{2}+\beta_{3}\right)}{\mu}\left(\frac{d u}{d x}\right)^{2} \frac{d^{2} u}{d x^{2}}-\frac{\sigma B_{0}^{2}}{\mu} u=0$

and the boundary conditions are

$u=0 \quad$ at $\quad x=0$,

$u=U \quad$ at $\quad x=d$.

Introducing the following non-dimensional parameters

$$
x^{\mathrm{m}}=\frac{x}{d}, \quad u^{\mathrm{m}}=\frac{u}{U}, \beta_{2}^{\mathrm{m}}=\frac{\beta_{2}}{\mu d^{2} / U^{2}}, m^{\mathrm{m} 2}=\frac{\sigma B_{\mathrm{o}}^{2}}{\mu / d^{2}}, \beta_{3}^{\mathrm{m}}=\frac{\beta_{3}}{\mu d^{2} / U^{2}},
$$

and dropping the ' $m$ ' notation (for simplicity), the boundary value problem (22) becomes

$$
\frac{d^{2} u}{d x^{2}}+6 \beta\left(\frac{d u}{d x}\right)^{2} \frac{d^{2} u}{d x^{2}}-m^{2} u=0
$$


with the boundary conditions

$u(x)=0$, at $x=0$,

$u(x)=1, \quad$ at $\quad x=1$,

where $\beta=\beta_{2}+\beta_{3}$.

\section{Solution of the problems in fuzzy environment}

To handle these problems we have taken TFNs and discretization in the form of $(\delta, \chi, \eta)$ and $(d, e, f)$ for the fuzzy parameters. This discretization is used in the boundary of the parallel plates for certain flow behavior because of the boundary is taken as fuzzified. The governing equation (24) with boundary conditions (25) are converted into FDEs as given below

$$
\begin{aligned}
& \frac{d^{2} \bar{u}(x ; \alpha)}{d x^{2}}+6 \text { e } \beta \text { e }\left(\frac{d \bar{u}(x ; \alpha)}{d x}\right)^{2} \text { e } \frac{d^{2} \bar{u}(x ; \alpha)}{d x^{2}}-m^{2} \text { e } \bar{u}(x ; \alpha)=0, \\
& \bar{u}(x ; \alpha)=\left[\frac{\alpha-1}{100}, \frac{1-\alpha}{100}\right] \text { at } x=0, \\
& \bar{u}(x ; \alpha)=\left[\frac{\alpha-1}{50}, \frac{1-\alpha}{50}\right] \text { at } x=1,
\end{aligned}
$$

where operator e defines the multiplication of fuzzy numbers with a real number and $\bar{u}(x ; \alpha)=[v(x ; \alpha), u(x ; \alpha)], 0 \leq \alpha \leq 1$, is the fuzzy velocity profile. $\frac{d \bar{u}(x ; \alpha)}{d x}$ and $\frac{d^{2} \bar{u}(x ; \alpha)}{d x^{2}}$ represent the fuzzy first and second order derivatives of fuzzy-valued function $\bar{u}(\mathrm{x} ; \alpha)$. $v(x ; \alpha)$ and $u(x ; \alpha)$ are lower and upper fuzzy velocity profiles, while boundary conditions in (27) are fuzzy boundary conditions [29]. So, Eqs. (26) and (27) in the form of lower and upper fuzzy boundary value problems are

$$
\frac{d^{2} v(x ; \alpha)}{d x^{2}}+6 \beta\left(\frac{d v(x ; \alpha)}{d x}\right)^{2} \frac{d^{2} v(x ; \alpha)}{d x^{2}}-m^{2} v(x ; \alpha)=0
$$




$$
\begin{aligned}
& v(x ; \alpha)=\frac{\alpha-1}{100} \quad \text { at } x=0, \\
& v(x ; \alpha)=\frac{13 \alpha-2}{200} \text { at } x=1, \\
& \frac{d^{2} u(x ; \alpha)}{d x^{2}}+6 \beta\left(\frac{d u(x ; \alpha)}{d x}\right)^{2} \frac{d^{2} u(x ; \alpha)}{d x^{2}}-m^{2} u(x ; \alpha)=0, \\
& u(x ; \alpha)=\frac{1-\alpha}{100} \quad \text { at } x=0, \\
& u(x ; \alpha)=\frac{9-5 \alpha}{80} \text { at } x=1 .
\end{aligned}
$$

Now we use the ADM in fuzzy boundary value problems (28) - (31). Eq. (28) in operator form becomes

$$
L_{1} v(x ; \alpha)=m^{2} v-6 \beta\left(\frac{d v(x ; \alpha)}{d x}\right)^{2} \frac{d^{2} v(x ; \alpha)}{d x^{2}},
$$

where $L_{1}=d^{2} / d x^{2}$ and the inverse operator is $\hat{L}^{-1}=\iint(\cdot) d x d x$. Applying $\hat{L}^{-1}$ on both sides of Eq. (32) yields

$$
v(x ; \alpha)=c_{1} x+c_{2}-6 \beta \hat{L}^{-1}\left(\frac{d v(x ; \alpha)}{d x}\right)^{2} \frac{d^{2} v(x ; \alpha)}{d x^{2}}+\hat{L}^{-1}\left[m^{2} v(x ; \alpha)\right],
$$

where the constants of integration are $c_{1}$ and $c_{2}$.

In view of Eqs. (16) and (17), Eq. (33) provides

$$
\sum_{n=0}^{\infty} v_{n}(x ; \alpha)=c_{1} x+c_{2}-6 \beta \hat{L}^{-1}\left(\frac{d v(x ; \alpha)}{d x}\right)^{2} \frac{d^{2} v(x ; \alpha)}{d x^{2}}+\hat{L}^{-1}\left[m^{2} v(x ; \alpha)\right] \text {. }
$$

We identify the zeroth component as

$$
v_{0}(x ; \alpha)=c_{1} x+c_{2},
$$

and the remaining components as the recurrence relation,

$$
v_{n+1}(x ; \alpha)=-6 \beta \hat{L}^{-1}\left[A_{n}^{*}(x ; \alpha)\right]+\hat{L}^{-1}\left[m^{2} v_{n}(x ; \alpha)\right] .
$$

where $A_{n}^{*}$, s represent the non-linear term in Eq. (34), and we give few $A_{n}^{*}, \mathrm{~s}$ as follows, which are defined in [20-21]: 


$$
\begin{aligned}
& A_{0}^{*}(x ; \alpha)= \frac{d^{2} v_{0}(x ; \alpha)}{d x^{2}}\left(\frac{d v_{0}(x ; \alpha)}{d x}\right)^{2} \\
& A_{1}^{*}(x ; \alpha)=\left(\frac{d v_{0}(x ; \alpha)}{d x}\right)^{2} \frac{d^{2} v_{1}(x ; \alpha)}{d x^{2}}+2 \frac{d v_{0}(x ; \alpha)}{d x} \frac{d v_{1}(x ; \alpha)}{d x} \frac{d^{2} v_{0}(x ; \alpha)}{d x^{2}}, \\
& A_{2}^{*}(x ; \alpha)=\left(\frac{d v_{1}(x ; \alpha)}{d x}\right)^{2} \frac{d^{2} v_{0}(x ; \alpha)}{d x^{2}}+2 \frac{d v_{0}(x ; \alpha)}{d x} \frac{d v_{2}(x ; \alpha)}{d x} \frac{d^{2} v_{0}(x ; \alpha)}{d x^{2}}+2 \frac{d v_{0}(x ; \alpha)}{d x} \frac{d v_{1}(x ; \alpha)}{d x} \frac{d^{2} v_{1}(x ; \alpha)}{d x^{2}} \\
&+\left(\frac{d v_{0}(x ; \alpha)}{d x}\right)^{2} \frac{d^{2} v_{2}(x ; \alpha)}{d x^{2}}, \\
& \mathrm{M}
\end{aligned}
$$

The corresponding fuzzy boundary conditions become,

$$
\begin{aligned}
& v_{0}(x ; \alpha)=\frac{\alpha-1}{100}, \quad \text { at } x=0 \\
& v_{0}(x ; \alpha)=\frac{13 \alpha-2}{200}, \text { at } x=1, \\
& v_{n}(x ; \alpha)=0 \text { at } x=0, \\
& v_{n}(x ; \alpha)=0 \text { at } x=1, \quad n>0 .
\end{aligned}
$$

Solving Eqs. (35) to (39) by using $A_{n}^{*}$, s from Eq. (37), we have the values of $v_{0}(x ; \alpha), v_{1}(x ; \alpha)$ and $v_{2}(x ; \alpha)$, after using these values in Eq. (34), we find the following expression of lower fuzzy velocity profile

$$
v(x ; \alpha)=A_{1} x+A_{4}+\frac{m^{2}}{6}\left(A_{1} x^{3}+3 A_{4} x^{2}-A_{2} x\right)\left(1-\beta A_{1}^{2}\right)+\frac{m^{4}}{360}\left(3 A_{1} x^{5}+15 A_{4} x^{4}-10 A_{2} x^{3}+A_{3} x\right) .
$$

Similarly, we can find the upper velocity profile as follows

$$
u(x ; \alpha)=B_{1} x+B_{4}+\frac{m^{2}}{6}\left(B_{1} x^{3}+3 B_{4} x^{2}-B_{2} x\right)\left(1-\beta B_{1}^{2}\right)+\frac{m^{4}}{360}\left(3 B_{1} x^{5}+15 B_{4} x^{4}-10 B_{2} x^{3}+B_{3} x\right) .
$$

Eqs. (41) and (42) indicates the lower and upper velocity profiles for the MHD flow of a nonNewtonian third grade fluid between two parallel plates. In these solutions $\beta$ is the nonNewtonian fluid parameter. By setting $\beta=0$ we recover the same solutions for the viscous fluid.

\subsection{Plane Poiseuille flow}

We consider the steady laminar flow of third grade fluid between two stationary infinite parallel plats under a constant pressure gradient. The separation between two plates is $2 \mathrm{~d}$ and the 
plates are at $x=-d$ and $x=d$ (see in the Fig. 2). Thus, the governing equation (12) with the transversal magnetic field and constant pressure gradient takes the following form

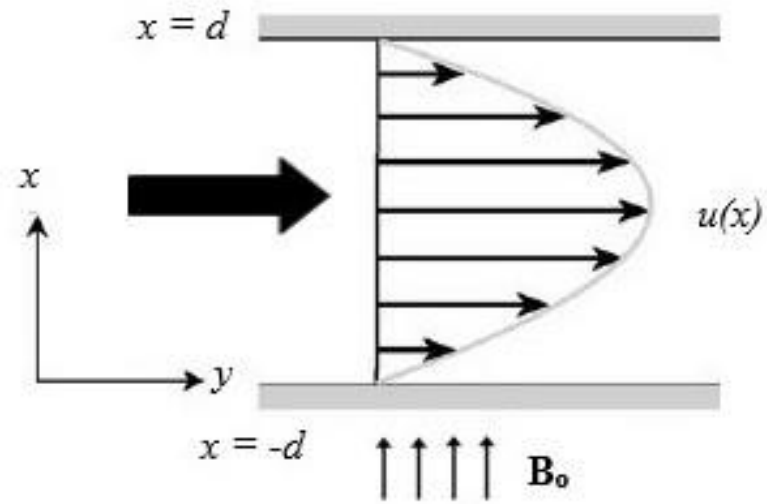

Fig. 2. Geometry of the Poiseuille flow.

$\frac{d^{2} u}{d x^{2}}+\frac{6\left(\beta_{3}+\beta_{2}\right)}{\mu}\left(\frac{d u}{d x}\right)^{2} \frac{d^{2} u}{d x^{2}}-\frac{\sigma B_{0}^{2}}{\mu} u=\frac{1}{\mu} \frac{d p^{ \pm}}{d y}$,

with the boundary conditions

$\begin{array}{ll}u(x)=0 & \text { at } \quad x=d, \\ u(x)=0 & \text { at } \quad x=-d .\end{array}$

Presenting the following non-dimensional parameters

$\beta_{2}^{\mathrm{m}}=\frac{\beta_{2}}{\mu d^{2} / U^{2}}, \beta_{3}^{\mathrm{m}}=\frac{\beta_{3}}{\mu d^{2} / U^{2}}, x^{\mathrm{m}}=\frac{x}{d}, p^{\mathrm{m}}=\frac{p}{\mu U / d}, m^{\mathrm{m} 2}=\frac{\sigma B_{0}^{2}}{\mu / d^{2}}, u^{\mathrm{m}}=\frac{u}{U}, y^{\mathrm{m}}=\frac{y}{d}$,

into Eqs. (43) and (44), after dropping ' $\mathrm{m}$ ' we have

$\frac{d^{2} u}{d x^{2}}+6 \beta\left(\frac{d u}{d x}\right)^{2} \frac{d^{2} u}{d x^{2}}-m^{2} u=\frac{d p}{d y}$,

with the boundary conditions

$u(x)=0 \quad$ at $\quad x=1$,

$u(x)=0 \quad$ at $\quad x=-1$.

To solve the above boundary value problem, we adopt the same procedure as in sec 4.1 . For this Eqs. (46) and (47) are converted into FDE of the following form 
$\frac{d^{2} \bar{u}(x ; \alpha)}{d x^{2}}+6$ e $\beta$ e $\left(\frac{d \bar{u}(x ; \alpha)}{d x}\right)^{2}$ e $\frac{d^{2} \bar{u}(x ; \alpha)}{d x^{2}}-m^{2}$ e $\bar{u}(x ; \alpha)=\frac{d p}{d y}$,

with the fuzzy boundary conditions

$$
\begin{aligned}
& \bar{u}(x ; \alpha)=\left[\frac{\alpha-1}{100}, \frac{1-\alpha}{100}\right] \quad \text { at } \quad x=1, \\
& \bar{u}(x ; \alpha)=\left[\frac{\alpha-1}{50}, \frac{1-\alpha}{50}\right] \quad \text { at } \quad x=-1 .
\end{aligned}
$$

Eqs. (48) and (49) in the form of lower and upper fuzzy boundary value problems are

$$
\begin{aligned}
& \frac{d^{2} v(x ; \alpha)}{d x^{2}}+6 \beta\left(\frac{d v(x ; \alpha)}{d x}\right)^{2} \frac{d^{2} v(x ; \alpha)}{d x^{2}}-m^{2} v(x ; \alpha)=\frac{d p}{d y}, \\
& v(x ; \alpha)=\frac{\alpha-1}{100} \quad \text { at } x=1, \\
& v(x ; \alpha)=\frac{2 \alpha-2}{100} \text { at } x=-1, \\
& \frac{d^{2} u(x ; \alpha)}{d x^{2}}+6 \beta\left(\frac{d u(x ; \alpha)}{d x}\right)^{2} \frac{d^{2} u(x ; \alpha)}{d x^{2}}-m^{2} u(x ; \alpha)=\frac{d p}{d y}, \\
& u(x ; \alpha)=\frac{1-\alpha}{100} \quad \text { at } x=1, \\
& u(x ; \alpha)=\frac{2-2 \alpha}{100} \text { at } x=-1 .
\end{aligned}
$$

Now we use the ADM in fuzzy boundary value problems (50) - (53). Eq. (50) in the operator form becomes

$$
L_{1} v(x ; \alpha)=\frac{d p}{d y}+m^{2} v(x ; \alpha)-6 \beta\left(\frac{d v(x ; \alpha)}{d x}\right)^{2} \frac{d^{2} v(x ; \alpha)}{d x^{2}},
$$

where $L_{1}=d^{2} / d x^{2}$ and inverse operator is $\hat{L}^{-1}=\iint(\cdot) d x d x$. Applying $\hat{L}^{-1}$ on both sides of Eq.

(54) we get

$$
v(x ; \alpha)=c_{1} x+c_{2}-6 \beta \hat{L}^{-1}\left(\frac{d v(x ; \alpha)}{d x}\right)^{2} \frac{d^{2} v(x ; \alpha)}{d x^{2}}+\hat{L}^{-1}\left[m^{2} v(x ; \alpha)\right]+\hat{L}^{-1}\left[\frac{d p}{d y}\right],
$$

where the constants of integration are $c_{1}$ and $c_{2}$. 
In view of Eqs. (16) and (17), Eq. (55) provides

$$
\sum_{n=0}^{\infty} v_{n}(x ; \alpha)=c_{1} x+c_{2}+\hat{L}^{-1}\left[\frac{d p}{d y}\right]-6 \beta \hat{L}^{-1}\left(\frac{d v(x ; \alpha)}{d x}\right)^{2} \frac{d^{2} v(x ; \alpha)}{d x^{2}}+\hat{L}^{-1}\left[m^{2} v(x ; \alpha)\right]
$$

Consequently, the decomposition method yields the recurrence relation,

$$
\begin{aligned}
& v_{0}(x ; \alpha)=c_{1} x+c_{2}+\hat{L}^{-1}\left(\frac{d p}{d y}\right), \\
& v_{n+1}(x ; \alpha)=-6 \beta \hat{L}^{-1}\left[A_{n}^{*}(x ; \alpha)\right]+\hat{L}^{-1}\left[m^{2} v_{n}(x ; \alpha)\right],
\end{aligned}
$$

The corresponding fuzzy boundary conditions, become

$$
\begin{aligned}
& v_{0}(x ; \alpha)=\frac{\alpha-1}{100} \quad \text { at } x=1, \\
& v_{0}(x ; \alpha)=\frac{2 \alpha-2}{100} \text { at } x=-1, \\
& v_{n}(x ; \alpha)=0 \text { at } x=1, \\
& v_{n}(x ; \alpha)=0 \text { at } x=-1, \quad n>0 .
\end{aligned}
$$

Solving Eqs. (57) - (59) by using $A_{n}^{*}, \mathrm{~s}$ from Eq. (37), we have the values of $v_{0}(x ; \alpha), v_{1}(x ; \alpha)$ and $v_{2}(x ; \alpha)$, after using these values in Eq. (56), we find the following expression of lower fuzzy velocity profile

$$
\begin{aligned}
& v(x ; \alpha)=\frac{p x^{2}}{2}+D_{2} x+E_{2}-\frac{\beta\left(p x+D_{2}\right)^{4}}{2 p}-6 \beta G x+6 \beta F x+\frac{2 \beta^{2}}{p}\left(p x+D_{2}\right)^{6}+\frac{2 \beta}{p}\left(p x+D_{2}\right)^{3} \\
& +m^{2}\left[\frac{p x^{4}}{24}+\left(\frac{D_{2}-6 G \beta}{6}\right) x^{3}+\left(\frac{E_{2}+6 \beta F}{2}\right) x^{2}+\left(\frac{6 Z-D_{2}}{6}\right) x-\frac{p+12 E_{2}}{24}-\frac{\beta\left(p x+D_{2}\right)^{6}}{60 p^{3}}+V\right] \\
& -m^{2} \beta\left[\frac{p^{3} x^{6}}{6}+\left(\frac{7 p D_{2}^{2}+4 p E_{2}}{4}\right) x^{4}+\left(2 p E_{2} D_{2}+D_{2}^{3}+2 p^{2} E_{2}+p E_{2} D_{2}\right) x^{3}-\frac{D_{2}\left(p x+D_{2}\right)^{3}}{3 p}\right] \\
& +m^{4}\left[\frac{p x^{6}}{720}+\frac{D_{2} x^{5}}{120}+\frac{E_{2} x^{4}}{24}-\frac{D_{2} x^{3}}{36}-\left(\frac{p+12 E_{2}}{48}\right) x^{2}\right]-6 \beta W_{1} x-6 \beta W+\ldots
\end{aligned}
$$

Similarly, we can find the upper velocity profile as follows 


$$
\begin{aligned}
& u(x ; \alpha)=\frac{p x^{2}}{2}+D_{3} x+E_{3}-\frac{\beta\left(p x+D_{3}\right)^{4}}{2 p}-6 \beta G_{1} x+6 \beta F_{1} x+\frac{2 \beta^{2}}{p}\left(p x+D_{3}\right)^{6}+\frac{2 \beta}{p}\left(p x+D_{3}\right)^{3} \\
& +m^{2}\left[\frac{p x^{4}}{24}+\left(\frac{D_{3}-6 G_{1} \beta}{6}\right) x^{3}+\left(\frac{E_{3}+6 \beta F_{1}}{2}\right) x^{2}+\left(\frac{6 Z_{1}-D_{3}}{6}\right) x-\frac{p+12 E_{3}}{24}-\frac{\beta\left(p x+D_{3}\right)^{6}}{60 p^{3}}+V_{1}\right] \\
& +m^{2} \beta\left[\frac{p^{3} x^{6}}{6}+p^{2} D_{3} x^{5}+\left(\frac{7 p D_{3}^{2}+4 p E_{3}}{4}\right) x^{4}+\left(2 p E_{3} D_{3}+D_{3}^{3}+2 p^{2} E_{3}+p E_{3} D_{3}\right) x^{3}+3 E_{3} D_{3}^{2} x^{2}\right] \\
& \left.+m^{4}\left[\frac{p x^{6}}{720}+\frac{D_{3} x^{5}}{120}+\frac{D_{3} x^{4}}{24}-\frac{D_{3} x^{3}}{36}-\left(\frac{p+12 E_{3}}{48}\right) D_{3}\right)^{3}\right]-6 \beta W_{2} x-6 \beta W_{3}+\ldots
\end{aligned}
$$

\subsection{Generalized Couette Flow}

Again we consider the steady laminar flow of a third grade fluid between two infinite horizontal parallel plates at a distance $d$ apart under a constant pressure gradient. The lower plate is fixed while the upper plate at $x=d$ is moving with uniform velocity $\mathrm{U}$. The plates are nonconducting and a magnetic field is applied in the vertical upward direction. Let y-axis be taken normal direction to flow and $\mathrm{x}$-axis in the direction of flow (See in Fig. 3). The resulting non dimensional differential equation (46) with boundary conditions (25), under the transversal magnetic field and constant pressure gradient is

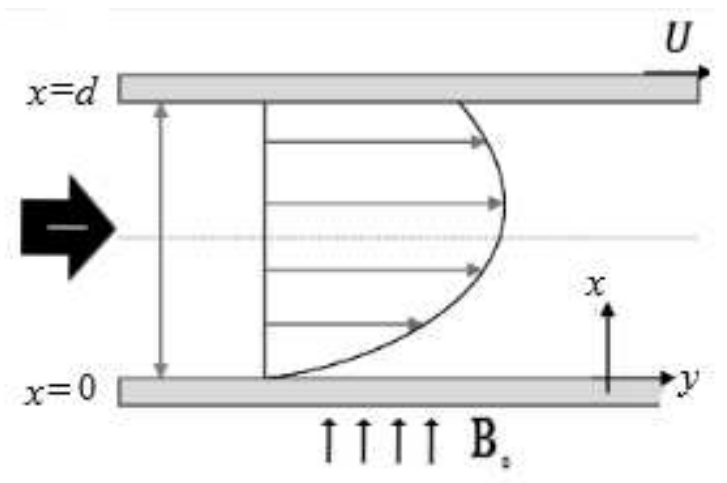

Fig. 3. Geometry of the generalized Couette flow. 
$\frac{d^{2} u}{d x^{2}}+6\left(\beta_{3}+\beta_{2}\right)\left(\frac{d u}{d x}\right)^{2} \frac{d^{2} u}{d x^{2}}-m^{2} u=\frac{d p}{d y}$,

$u(x)=0 \quad$ at $\quad x=0$,

$u(x)=1 \quad$ at $\quad x=1$.

To solve this boundary value problem, we assume the same procedure as in the previous sections, like Eqs. (64) and (65) are converted into FDE of the following form

$\frac{d^{2} \bar{u}(x ; \alpha)}{d x^{2}}+6$ e $\beta$ e $\left(\frac{d \bar{u}(x ; \alpha)}{d x}\right)^{2}$ e $\frac{d^{2} \bar{u}(x ; \alpha)}{d x^{2}}-m^{2}$ e $\bar{u}(x ; \alpha)=\frac{d p}{d y}$

with the fuzzy boundary conditions

$\bar{u}(x ; \alpha)=\left[\frac{\alpha-1}{100}, \frac{1-\alpha}{100}\right]$ at $x=0$,

$\bar{u}(x ; \alpha)=\left[\frac{\alpha-1}{50}, \frac{1-\alpha}{50}\right]$ at $x=1$,

Eqs. (66) and (67) in the form of lower and upper fuzzy boundary value problems are

$$
\begin{aligned}
& \frac{d^{2} v(x ; \alpha)}{d x^{2}}+6 \beta\left(\frac{d v(x ; \alpha)}{d x}\right)^{2} \frac{d^{2} v(x ; \alpha)}{d x^{2}}-m^{2} v(x ; \alpha)=\frac{d p}{d y}, \\
& v(x ; \alpha)=\frac{\alpha-1}{100} \quad \text { at } \quad x=0, \\
& v(x ; \alpha)=\frac{\alpha-1}{50} \quad \text { at } \quad x=1, \\
& \frac{d^{2} u(x ; \alpha)}{d x^{2}}+6 \beta\left(\frac{d u(x ; \alpha)}{d x}\right)^{2} \frac{d^{2} u(x ; \alpha)}{d x^{2}}-m^{2} u(x ; \alpha)=\frac{d p}{d y}, \\
& u(x ; \alpha)=\frac{1-\alpha}{100} \quad \text { at } x=0, \\
& u(x ; \alpha)=\frac{1-\alpha}{50} \quad \text { at } x=1 .
\end{aligned}
$$

Now we use the ADM in fuzzy boundary value problems (68) - (71). Adopting the same procedure as in sec 4.1, we find the following expression of lower and upper fuzzy velocity profiles, respectively 


$$
\begin{aligned}
& v(x ; \alpha)=\frac{p x^{2}}{2}+\frac{\beta}{2 p}\left[D^{4}-\left(D^{4}-(p+D)^{4}\right) x-(p x+D)^{4}\right]+\frac{6 \beta^{2}}{5 p}(p x+D)^{5}+\frac{6 \beta^{2}}{5 p}(p x+D)^{6}+D x \\
& +E+m^{2}\left[\begin{array}{l}
\left.-\frac{\beta p^{2}(2 p+3) x^{6}}{30}-\beta p^{2} D x^{5}+\frac{p-36 \beta p^{2} E-42 \beta p D^{2}}{24} x^{4}+\frac{D-24 \beta p E D-6 D^{3}}{6} x^{3}\right] \\
+\left[2 p E-12 \beta E D^{2}+\beta D^{4}\right) x^{2} \\
-\frac{\beta(p x+D)^{6}}{60 p^{3}}-\frac{\beta+4 D+12 E}{12 p}\left(D^{4}-(p+D)^{4}\right) x^{3}+R x+\frac{\beta D^{6}}{60 p^{3}}
\end{array}\right] \\
& +m^{4}\left[\frac{p x^{6}}{720}+\frac{D x^{5}}{120}+\frac{E x^{4}}{24}-\frac{p+4 D+12 E}{144} x^{3}\right]+\frac{3 \beta^{2}}{2 p^{2}}(p x+D)^{3}\left(D^{4}-(p+D)^{4}\right) \\
& -6 \beta L y-6 \beta K+\ldots,
\end{aligned}
$$

$$
\begin{aligned}
& \begin{array}{l}
u(x ; \alpha)=\frac{p x^{2}}{2}+D_{1} x+E_{1}+\frac{\beta}{2 p}\left[D_{1}^{4}-\left(D_{1}^{4}-\left(p+D_{1}\right)^{4}\right) x-\left(p x+D_{1}\right)^{4}\right]+\frac{6 \beta^{2}}{5 p}\left(p x+D_{1}\right)^{5} \\
-\frac{\beta p^{2}(2 p+3) x^{6}}{30}-\beta p^{2} D_{1} x^{5}+\frac{p-36 \beta p^{2} E_{1}-42 \beta p D_{1}^{2}}{24} x^{4} \\
+\frac{6 \beta^{2}}{5 p}\left(p x+D_{1}\right)^{6}+m^{2}\left[\begin{array}{l}
+\frac{D_{1}-24 \beta p E_{1} D_{1}-6 D_{1}^{3}}{6} x^{3}+\frac{\left(2 p E_{1}-12 \beta E_{1} D_{1}^{2}+\beta D_{1}^{4}\right) x^{2}}{4 p} \\
-\frac{p+4 D_{1}+12 E_{1}}{24} x+\frac{\beta}{12 p}\left(p+4 D_{1}+12 E_{1}\right)\left(p x+D_{1}\right)^{3} \\
-\frac{\beta\left(p x+D_{1}\right)^{6}}{60 p^{3}}-\frac{\beta}{12 p}\left(D_{1}^{4}-\left(p+D_{1}\right)^{4}\right) x^{3}+R_{1} x+\frac{\beta D_{1}^{6}}{60 p^{3}}
\end{array}\right] \\
+m^{4}\left[\frac{p x^{6}}{720}+\frac{D_{1} x^{5}}{120}+\frac{E_{1} x^{4}}{24}-\frac{p+4 D_{1}+12 E_{1}}{144} x^{3}\right]+\frac{3 \beta^{2}}{2 p^{2}}\left(p x+D_{1}\right)^{3}\left(D_{1}^{4}-\left(p+D_{1}\right)^{4}\right)
\end{array} \\
& \quad-6 \beta L_{1} x-6 \beta K_{1}+\ldots
\end{aligned}
$$

\section{Results and Discussion}

In this section we discuss graphically (plotted in Figs. 3-43) the effects of different flow parameters for different values of $\alpha$-cut $(\alpha=0,0.3,0.7,1)$ of the three basic flow problems in a fuzzy environment namely, plane Couette, plane Poiseuille, and generalized Couette flow of a third-grade fluid. Here, it may be observed that as $\alpha$ increases from 0 to 1 , we have a narrow 
width of fuzzy velocity profiles and uncertainty decreases drastically which finally provided crisp results (see Figs. 5, 9, 10, 17, 22, 23, 34, 38 and 39) for $\alpha=1$.

For plane Couette flow: Figs. 3-6 show the effect of magnetic parameter $m$ on the lower and upper bounds of velocity profiles under non-Newtonian fluid parameter $\beta$ at different values of fuzzy parameter $\alpha$-cut $(0 \leq \alpha \leq 1)$. The lower and upper bounds of velocity profiles decreases gradually near the center of the plates with increasing magnetic parameter $m$ under the generalization of the crisp velocity profile at different values of $\alpha$. Figs. 7-10 show the effect of $\beta$ on the lower and upper bounds of velocity profiles under magnetic parameter $m$ at different values of fuzzy parameter $\alpha$. The lower and upper bounds of velocity profiles increases gradually near the center of the plates with increasing non-Newtonian parameter $\beta$ at different values of fuzzy parameter $\alpha$. We can see from Figs. 6 and 10 that a classical solution or crisp solution of lower and upper bounds of velocity profiles are the same at $\alpha=1$. Fig. 11 describe the lower and upper bounds of fuzzy velocity profiles at the different values of $\alpha$. The crisp velocity profile is in between the lower and upper bounds of fuzzy velocity profiles so, for $\alpha=1$ the fuzzy velocity profile fall into classical velocity profile, which shows the present problem is a generalization of Kamran and Siddique [19]. Now for the membership function of TFNs, Figs.12 and 13 show the uncertain behavior in terms of the triangular fuzzy plot by different values of $m$ and $\beta$. In Fig. 12 the uncertain width gradually decreases with increasing input the magnetic parameter $m$ while Fig. 13 shows the uncertain width gradually increases with increasing $\beta$. Furthermore, it is seen that less width of fuzzy velocity profiles at the centre of the plates so the uncertainty is less than the boundary is less sensitive. While, width of fuzzy velocity profiles are greater so uncertainty is maximum then the boundary is more sensitive. Also it is observed that $v(\mathrm{x} ; \alpha)$ increases and $u(\mathrm{x} ; \alpha)$ decreases with increasing $\alpha$, hence solution is strong. When $\alpha$ increases the width between lower and upper bounds of fuzzy velocity profiles decreases and at $\alpha=1$ the projection of the peaks of the triangles coherent with the classical or crisp solution. Table 1, shows the analysis of lower, mid and upper bounds of velocity profiles at different values of $x$ with the fixed values of $\beta=0.75$ and $m=1$. The mid value of a TFN concurs with the crisp or classical value of the original problem. Furthermore fuzzy velocity profile always change with certain range for any fixed $\alpha$-cut and the range gradually decreases with increasing the values of $\alpha$-cut. 
For fully-developed plane Poiseuille flow: Figs. 14- 18 show the effect of $\beta$ on the lower and upper bounds of velocity profiles with constant pressure gradient at different values of fuzzy parameter $\alpha$. It is observed that the lower and upper bounds of velocity profiles decreases with increasing non-Newtonian parameter $\beta$. Figs. 19-23 show the effect of magnetic parameter $m$ on the lower and upper bounds of velocity profiles with constant pressure gradient at different values of $\alpha$. The lower and upper bounds of velocity profiles decreases with increasing magnetic parameter $m$. Figs. 18 and 23 provide a classical or crisp solutions that lower and upper bounds of velocity profiles are the same at $\alpha=1$. Fig. 24 describe the lower and upper bounds of fuzzy velocity profiles at the different values of $\alpha$. So, for $\alpha=1$ the fuzzy velocity profile fall into classical velocity profile, which shows the present problem is a generalization of Kamran and Siddique [19].

For the membership function of TFNs, Figs. 25-27 show the uncertain behavior in terms of the triangular fuzzy plot for different values of $m, d p / d y=p$ and $\beta$. In Figs. 25-26, the uncertain width gradually decreases with increasing input parameters $m$ and $\beta$ while, Fig. 27 shows the uncertain width rapidly increases with increasing the value of $p$. Furthermore, it is seen that less width of fuzzy velocity profiles at the centre of the plates so uncertainty is less than the boundary is less sensitive. While, width of fuzzy velocity profiles are greater so uncertainty is maximum then the boundary is more sensitive. Also it is observed that $v(\mathrm{x} ; \alpha)$ increases and $u(\mathrm{x} ; \alpha)$ decreases with increasing $\alpha$, hence solution is strong. When $\alpha$ increases the width between lower and upper bounds of fuzzy velocity profiles decreases and at $\alpha=1$ the projection of the peaks of the triangles coherent with the classical or crisp solution. Table 2, shows the analysis of lower, mid and upper bounds of velocity profiles at different values of $x$ with the fixed values of $\beta=0.3, \mathrm{p}=0.2$ and $m=0.2$. The mid value of a TFN concurs with the crisp or classical value of the original problem. Furthermore fuzzy velocity profile always change with certain range for any fixed $\alpha$-cut and the range gradually decreases with increasing the values of $\alpha$-cut.

For Generalized Couette Flow: Figs. 28-39 show the effect of fuzzy third grade fluid parameter $\beta$, magnetic parameter $m$ and $\alpha$ on the lower and upper velocity profiles with constant pressure gradient. In Figs. 28-31 the lower and upper velocity profiles decreases gradually with increasing non-Newtonian fuzzy parameter $\beta$. In Figs. 32-35 the lower and upper velocity profiles increases gradually with increasing magnetic parameter $m$. In Figs. 36-39 the lower and upper velocity 
profiles increases rapidly with increasing pressure gradient $p$. Figs. 32, 35 and 39 provides the crisp solutions that lower and upper velocity profiles are same at $\alpha=1$. Fig. 40 describes the lower and upper bounds of fuzzy velocity profiles at the different values of $\alpha$. The crisp velocity profile is in between the lower and upper bounds of fuzzy velocity profiles so, for $\alpha=1$ the fuzzy velocity profile fall into crisp or classical velocity profile, which shows the present problem is a generalization of Kamran and Siddique [19].

For the membership function of TFNs, Figs. 41-43 show the uncertain behavior in terms of the triangular fuzzy plot by different values of $m, p$ and $\beta$. In Figs. 41-42, the uncertain width gradually increases with increasing input parameters $m$ and $\beta$ while Fig. 43 shows the uncertain width rapidly increases with increasing the value of $d p / d y=p$. Furthermore, it is seen that less width of fuzzy velocity profiles at the centre of the plates so uncertainty is less than the boundary is less sensitive. While, width of fuzzy velocity profiles are greater so uncertainty is maximum then the boundary is more sensitive. Table 3 , shows the analysis of lower, mid and upper bounds of velocity profiles at different values of $x$ with the fixed values of $\beta=0.2, p=0.015$ and $m=0.1$. The mid value of TFN concurs with the crisp or classical value of the original problem. Furthermore fuzzy velocity profile always change with certain range for any fixed $\alpha$-cut and the range gradually decreases with increasing the values of $\alpha$-cut.

\section{Plane Couette Flow}

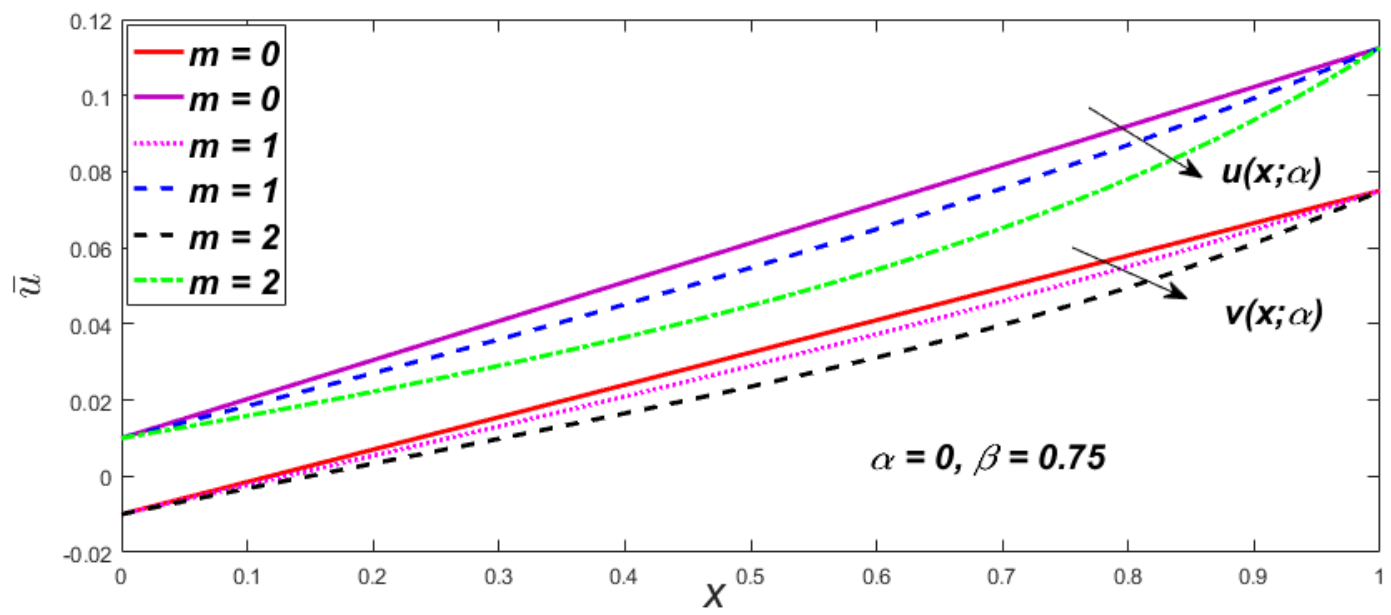

Fig. 3. Fuzzy velocity profiles for influence of $m$. 


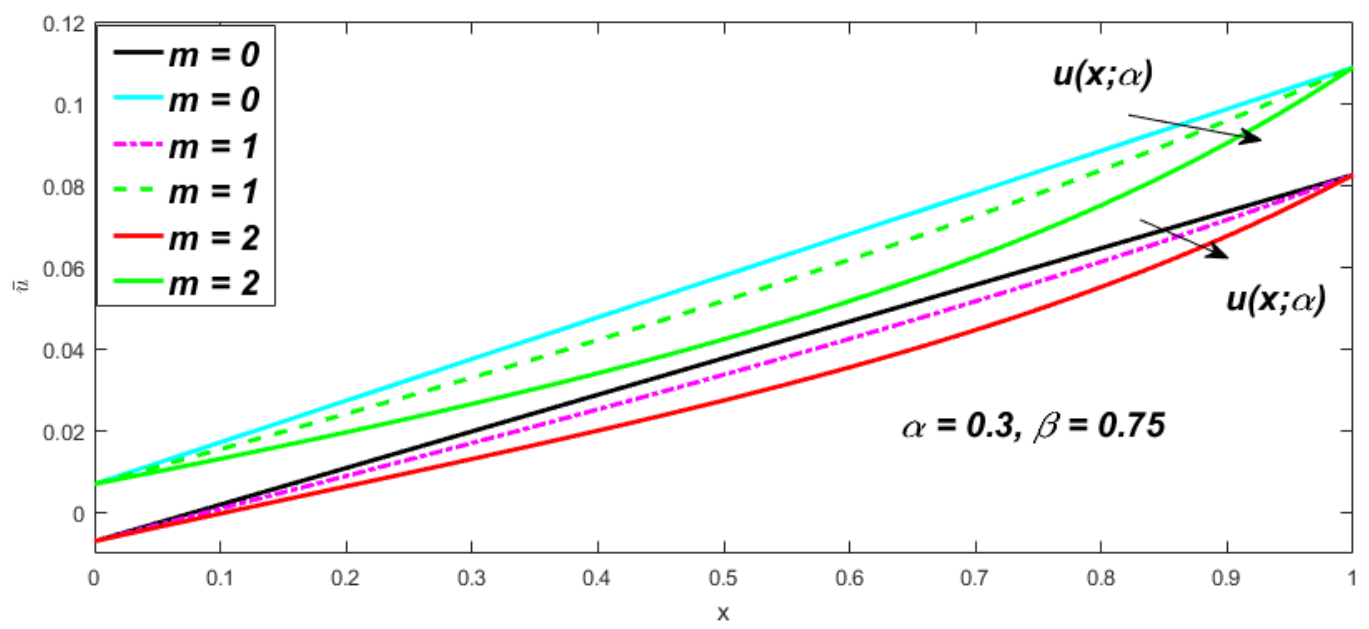

Fig. 4. Fuzzy velocity profiles for influence of $m$.

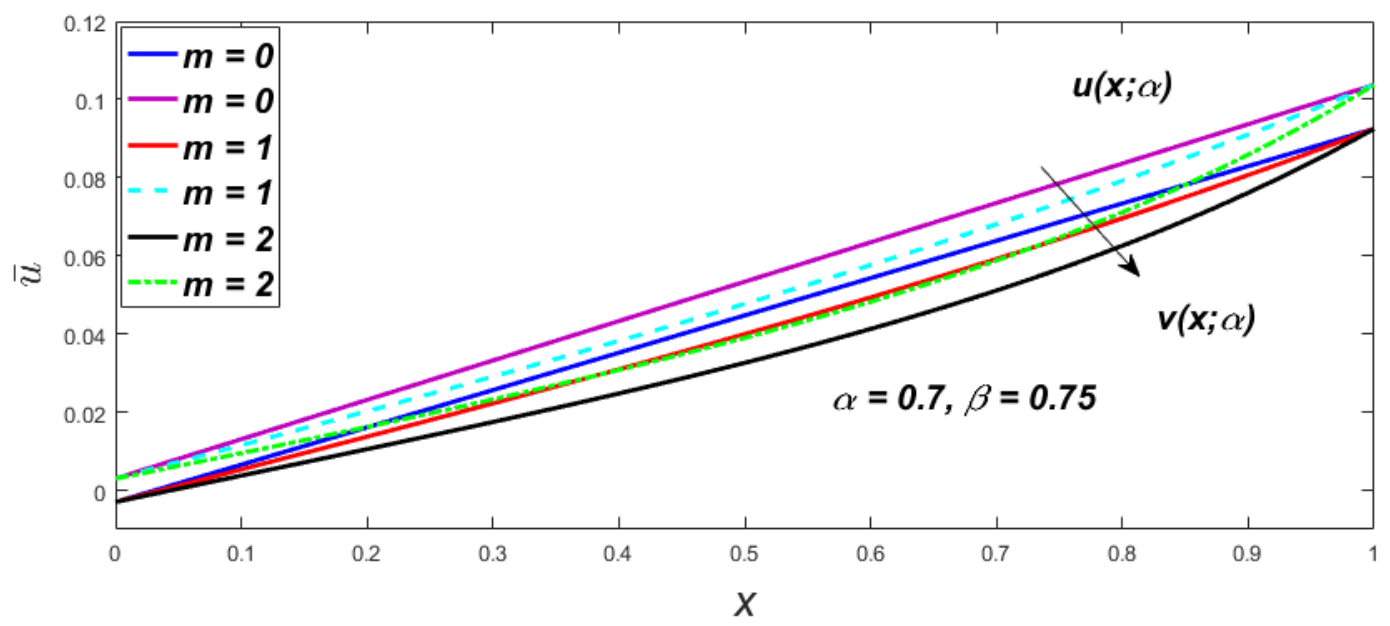

Fig. 5. Fuzzy velocity profiles for influence of $m$. 


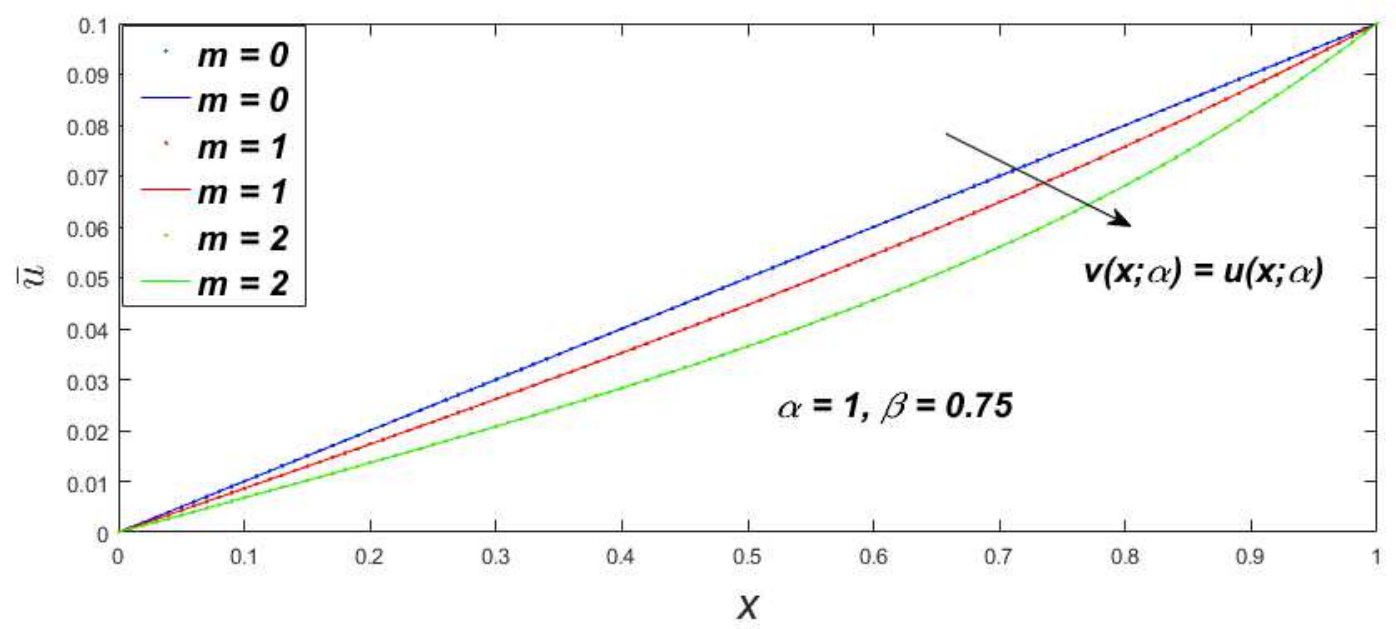

Fig. 6. Fuzzy velocity profiles for influence of $m$.

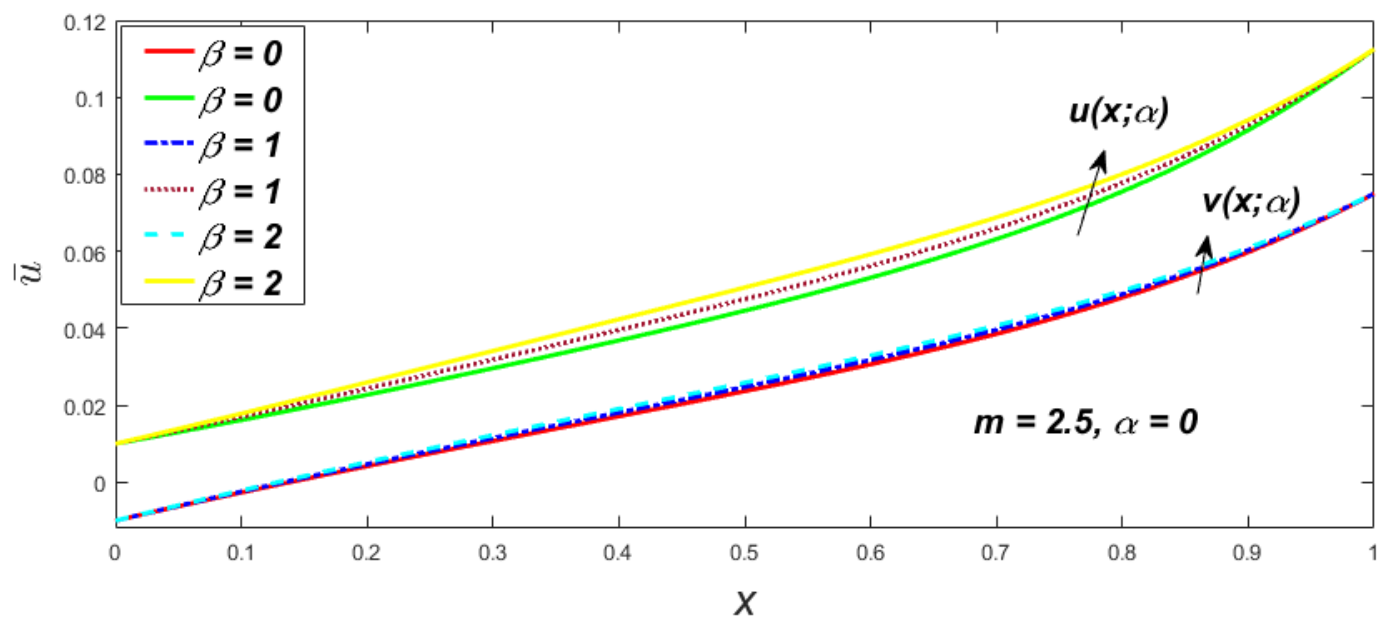

Fig. 7. Fuzzy velocity profiles for influence

of $\beta$. 


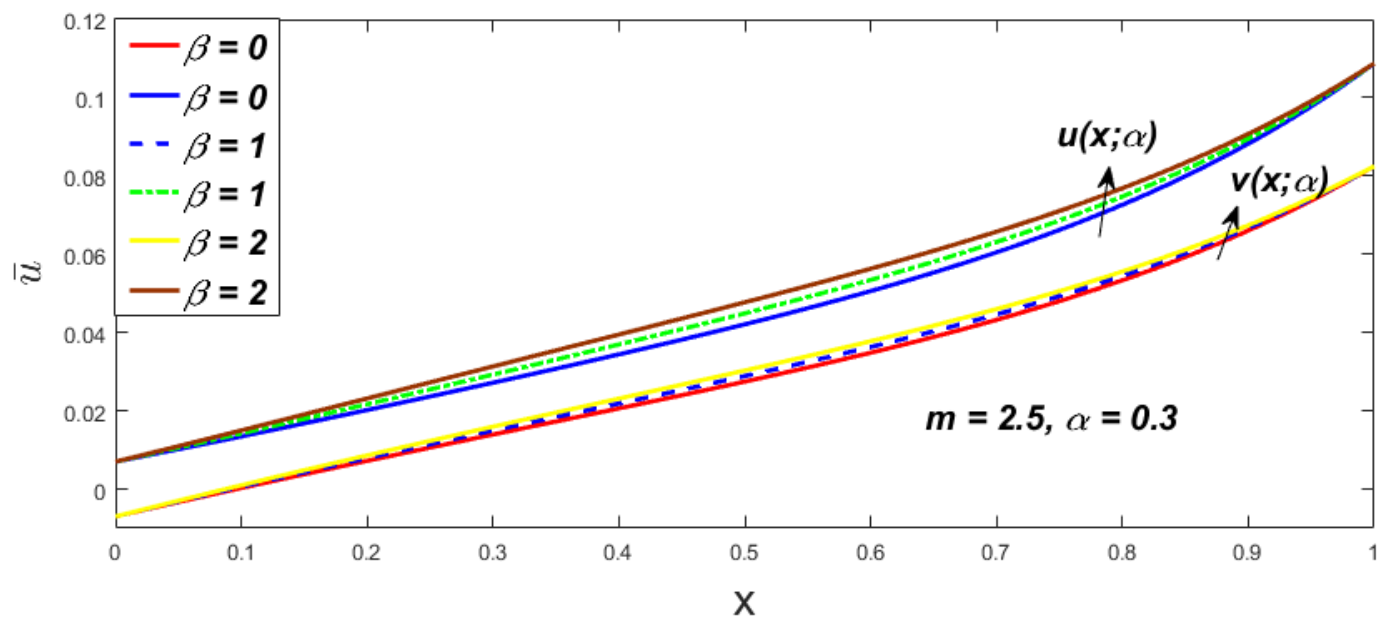

Fig. 8. Fuzzy velocity profiles for influence

of $\beta$.

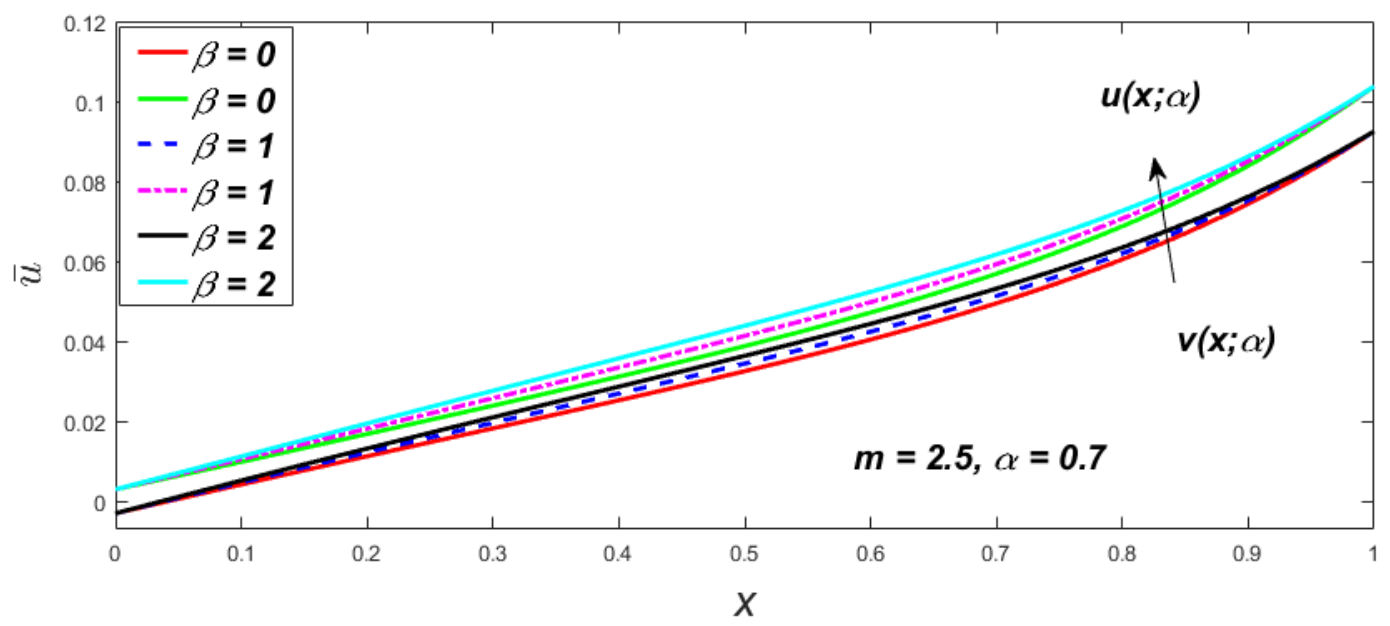

Fig. 9. Fuzzy velocity profiles for influence of $\beta$. 


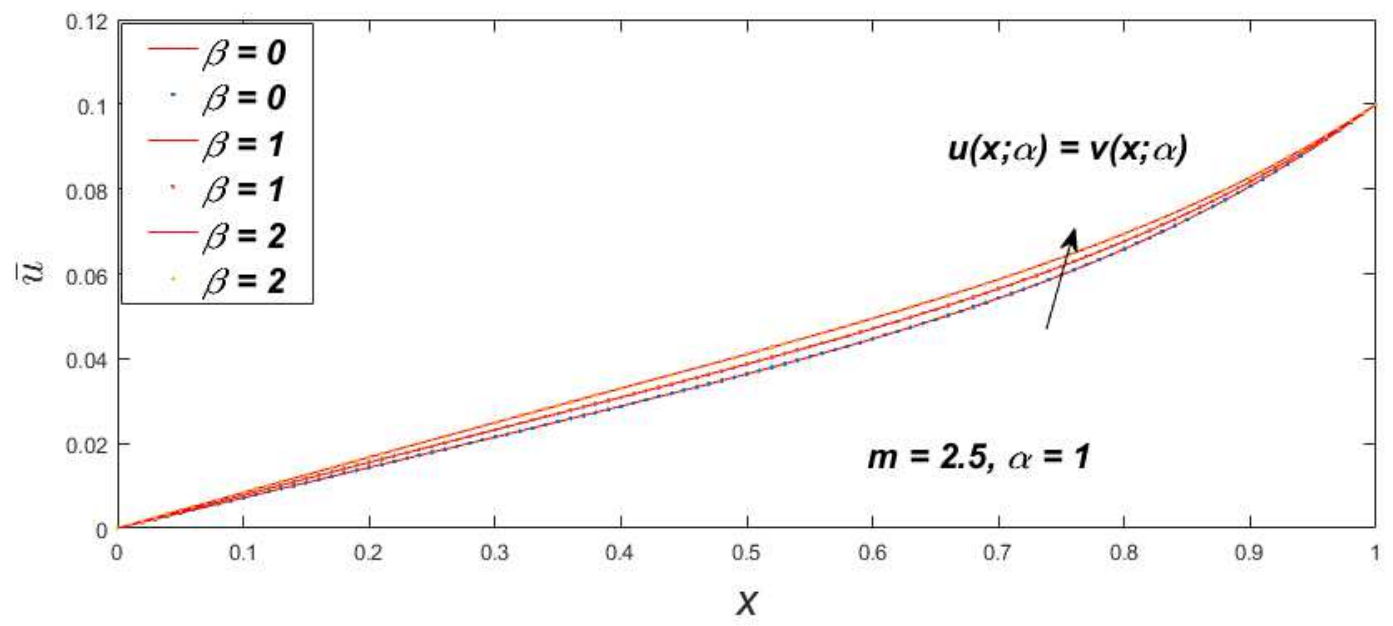

Fig. 10. Fuzzy velocity profiles for influence of $\beta$.

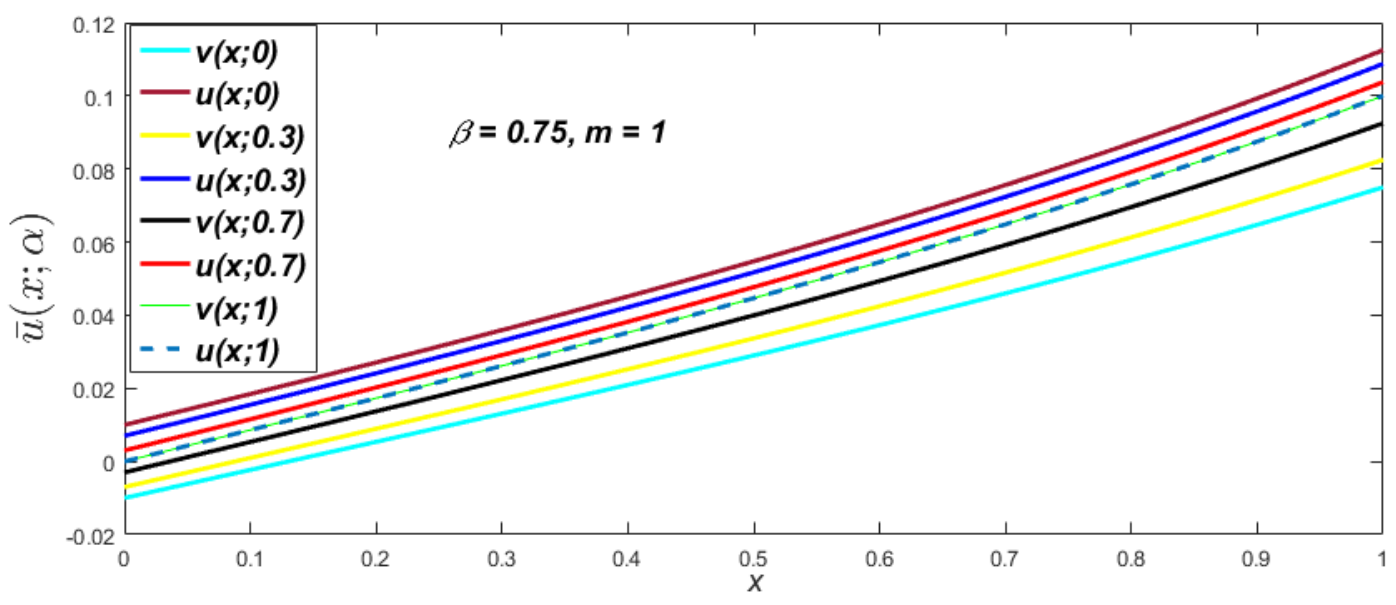

Fig. 11. Lower and upper bounds of velocity profiles for different values of $\alpha$-cut

$$
(0 \leq \alpha \leq 1) \text {. }
$$

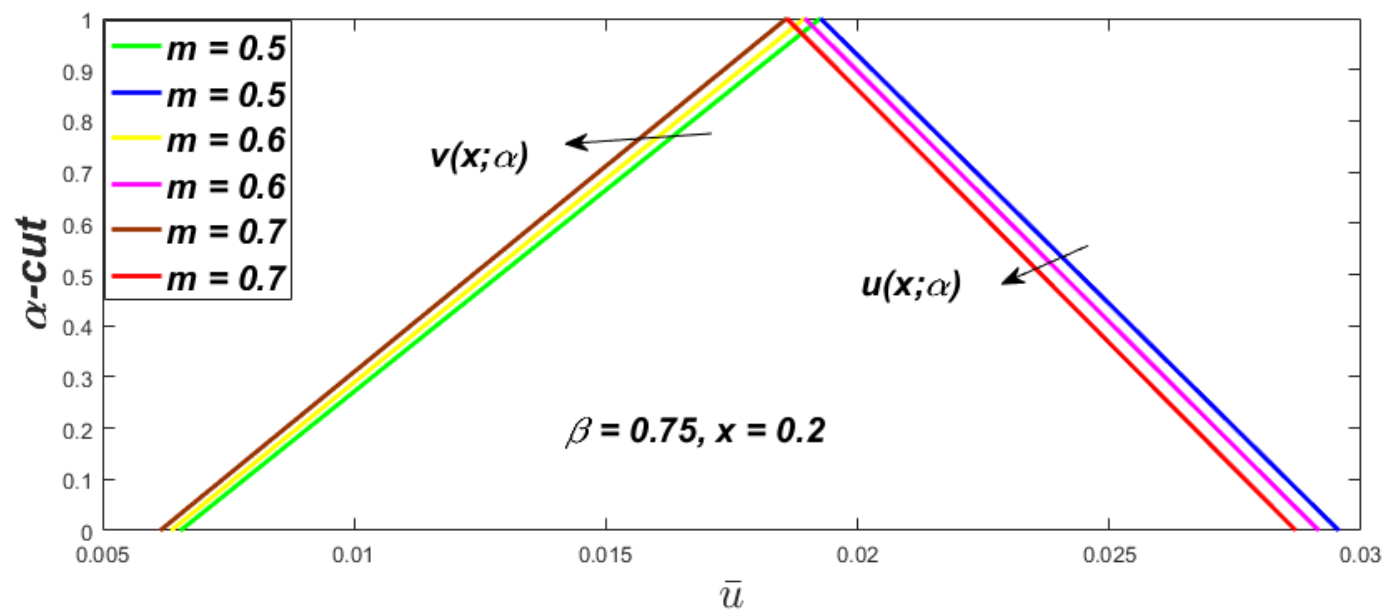

Fig. 12. Triangular membership function of lower and upper bounds of velocity profiles for influence of $m$. 


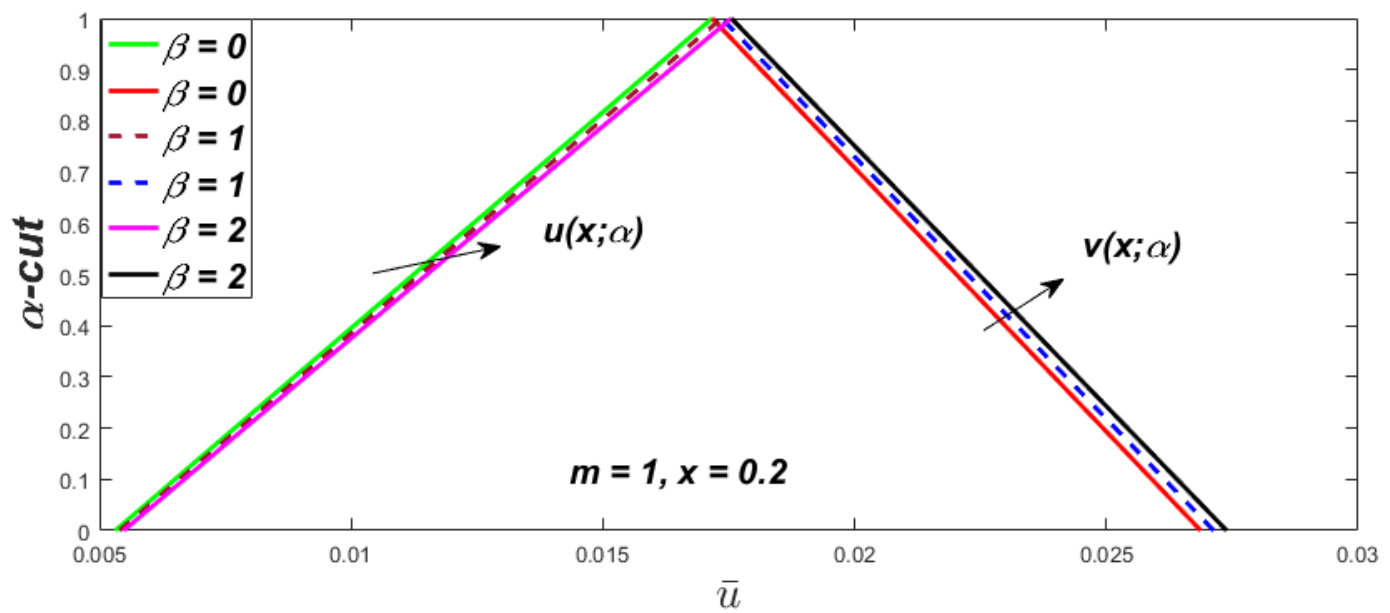

Fig. 13. Triangular membership function of lower and upper bounds of velocity profiles for influence of $\beta$.

Table 1. Fuzzy solution of lower, mid and upper bounds of velocity profiles at $\beta=0.75, m=1$ with different values of $\mathrm{x}$.

\begin{tabular}{|c|c|c|c|}
\hline $\mathrm{x}$ & $v(x ; \alpha)$ at $\alpha=0$ & Mid Value at $\alpha=0$ & $u(x ; \alpha)$ at $\alpha=0$ \\
\hline 0 & -0.010000000000000 & 0 & 0.010000000000000 \\
\hline 0.1 & -0.002299483093750 & 0.008073921533203 & 0.018447326160156 \\
\hline 0.2 & 0.005376984000000 & 0.016221659375000 & 0.027066334750000 \\
\hline 0.3 & 0.013102033552083 & 0.024518705615234 & 0.035935377678385 \\
\hline 0.4 & 0.020948997833333 & 0.033042427343750 & 0.045135856854167 \\
\hline 0.5 & 0.028992759114583 & 0.041873004150391 & 0.054753249186198 \\
\hline 0.6 & 0.037310599666667 & 0.051094365625000 & 0.064878131583333 \\
\hline 0.7 & 0.045983051760417 & 0.060795128857422 & 0.075607205954427 \\
\hline 0.8 & 0.055094747666667 & 0.071069535937500 & 0.087044324208333 \\
\hline 0.9 & 0.064735269656250 & 0.082018391455078 & 0.099301513253906 \\
\hline 1 & 0.075000000000000 & 0.0937500000000000 & 0.1125000000000000 \\
\hline
\end{tabular}




\section{Plane Poiseuille flow}

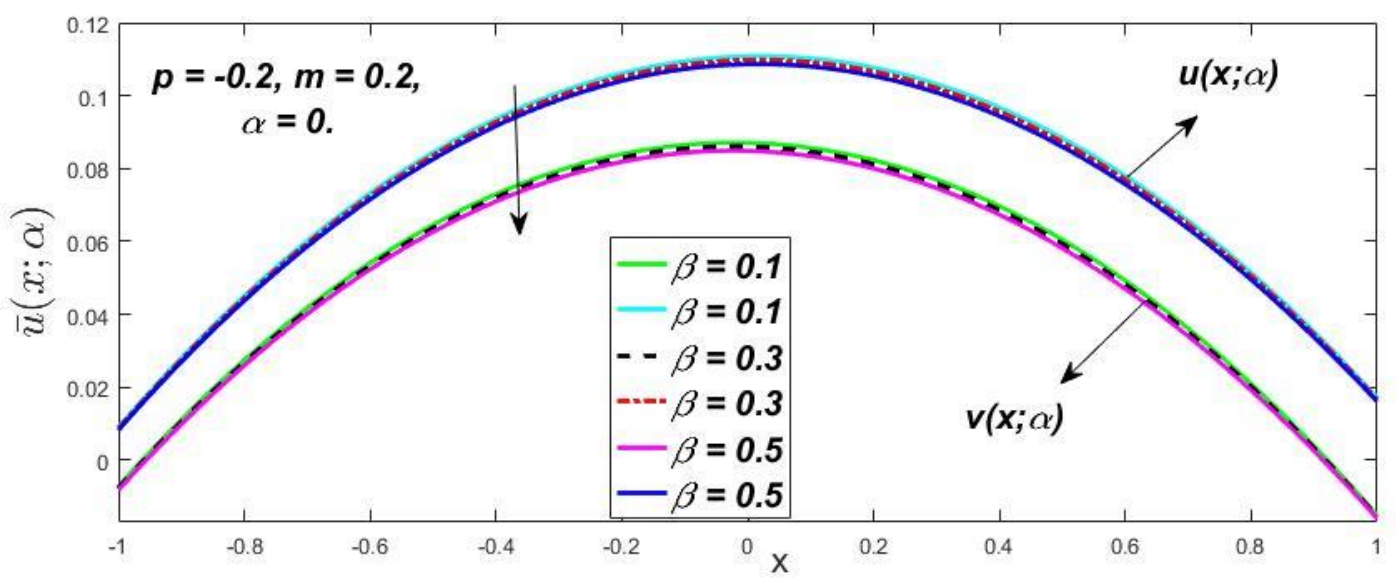

Fig. 14. Fuzzy velocity profiles for influence of $\beta$.

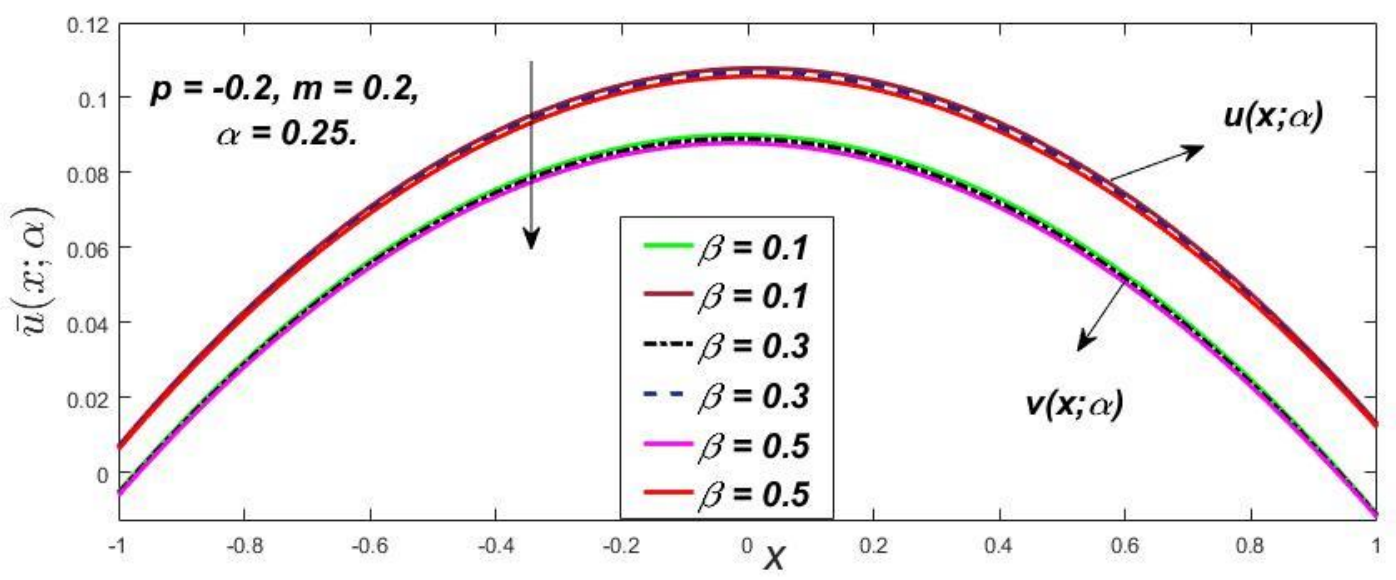

Fig. 15. Fuzzy velocity profiles for influence of $\beta$. 


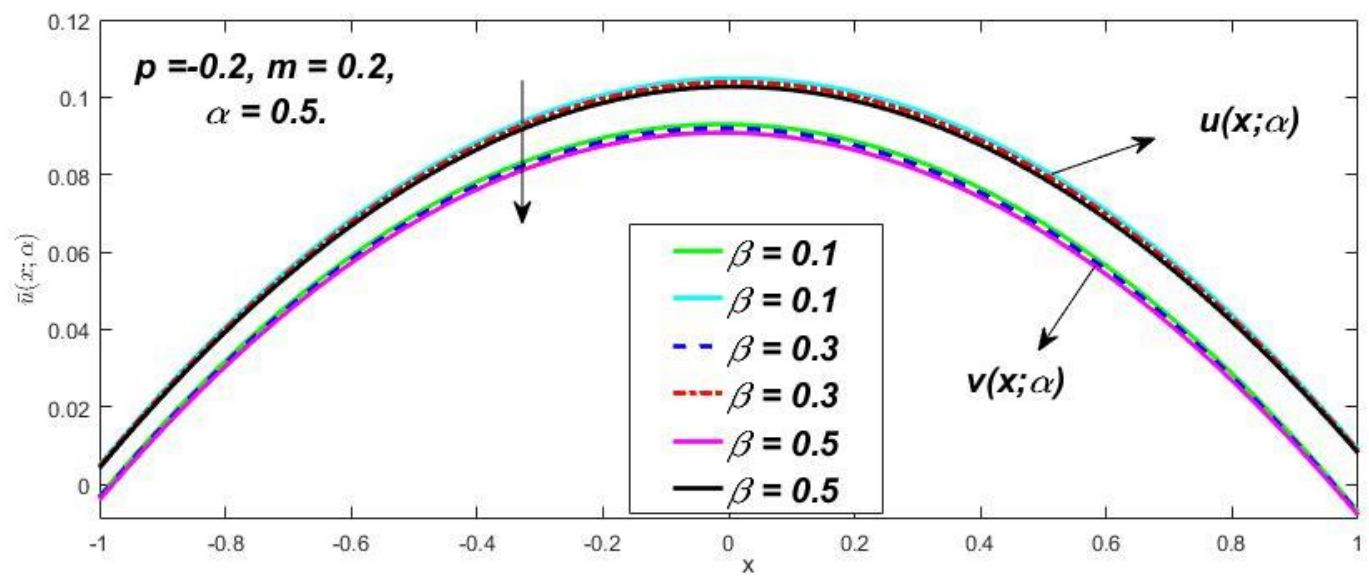

Fig. 16. Fuzzy velocity profiles for influence of $\beta$.

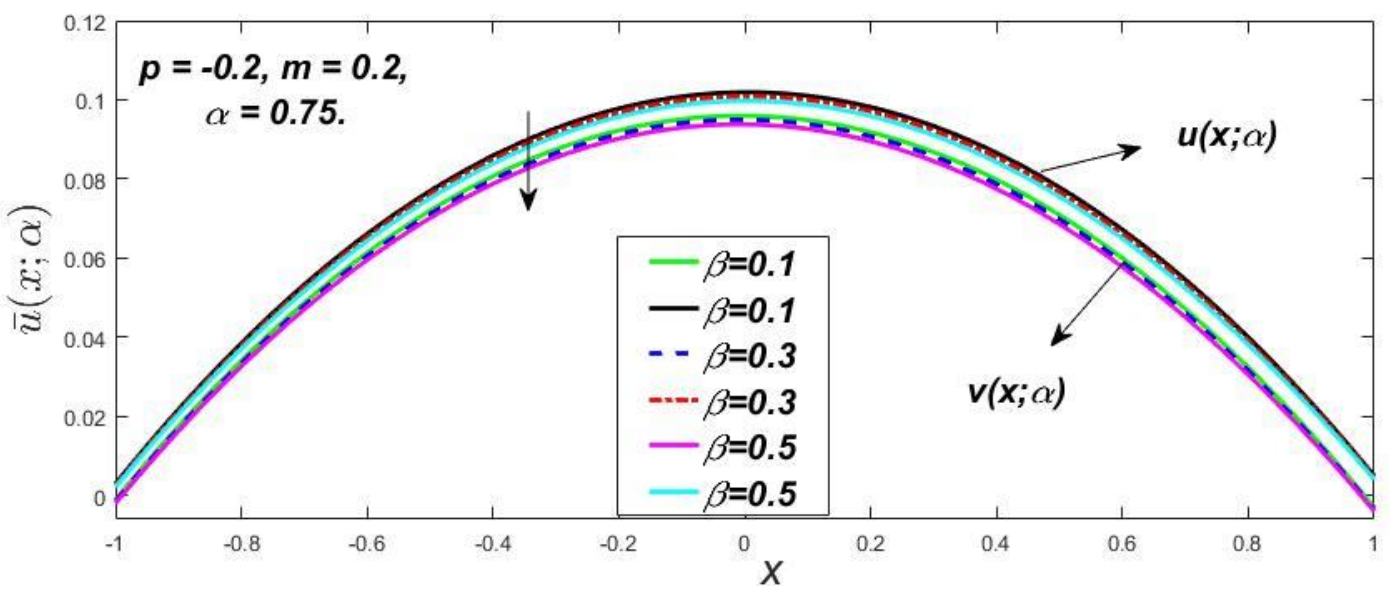

Fig. 17. Fuzzy velocity profiles for influence of $\beta$. 


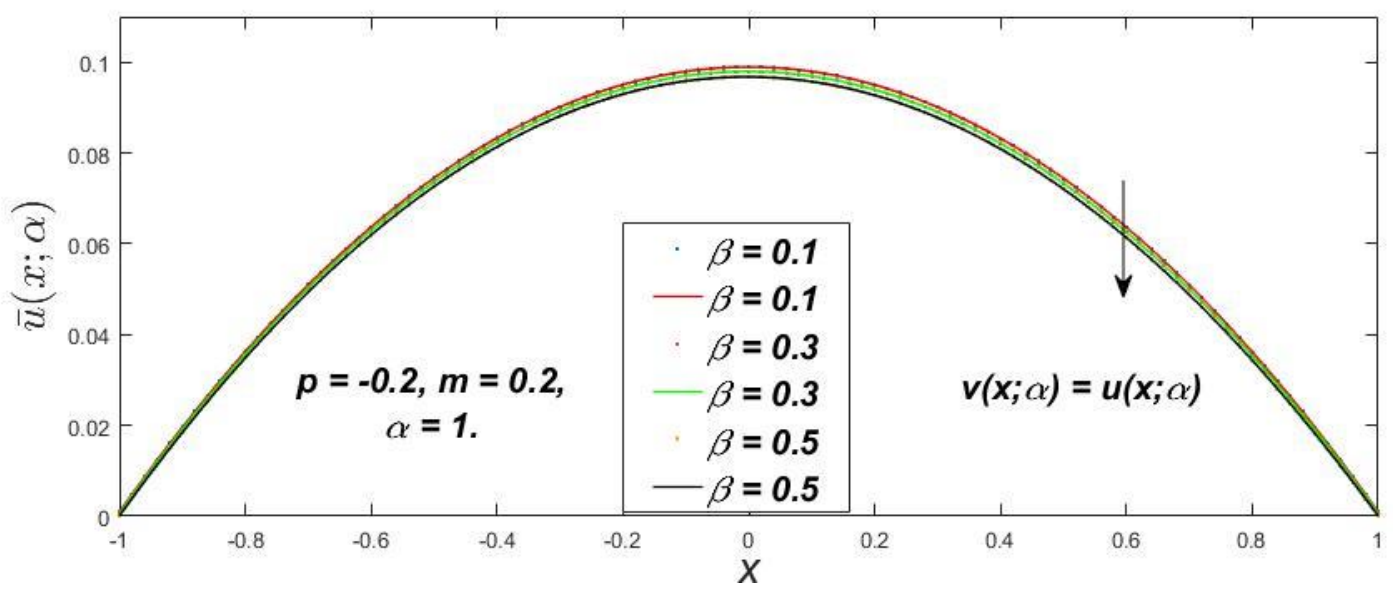

Fig. 18. Fuzzy velocity profiles for influence of $\beta$.

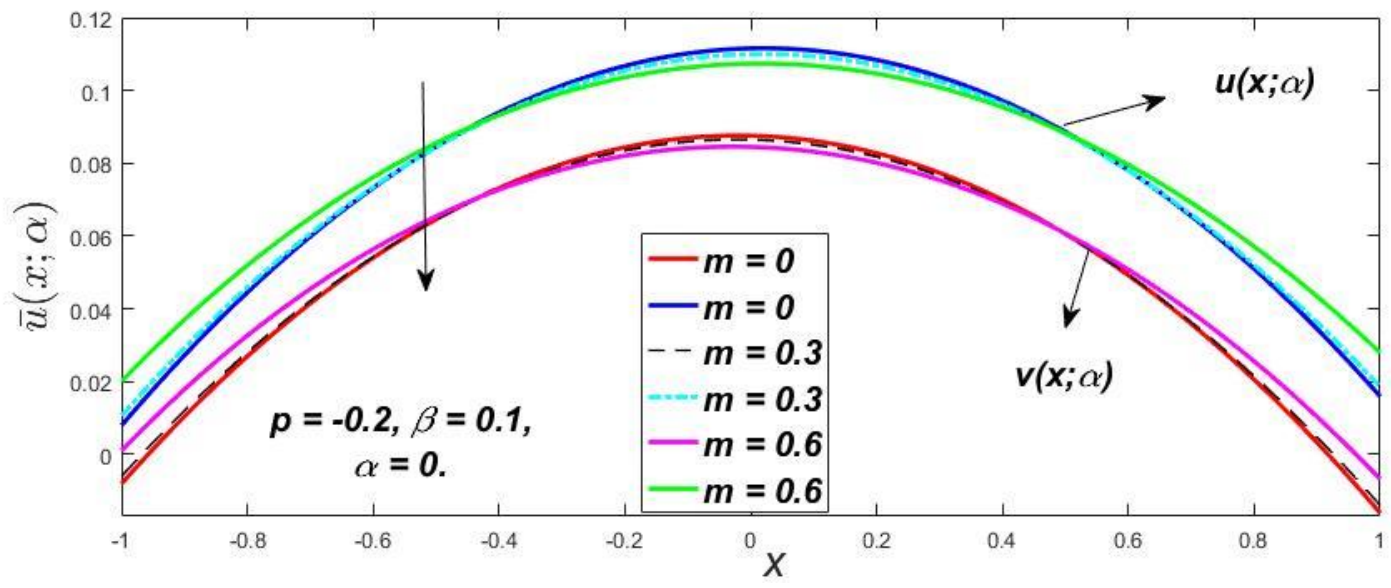

Fig. 19. Fuzzy velocity profiles for influence of $m$. 


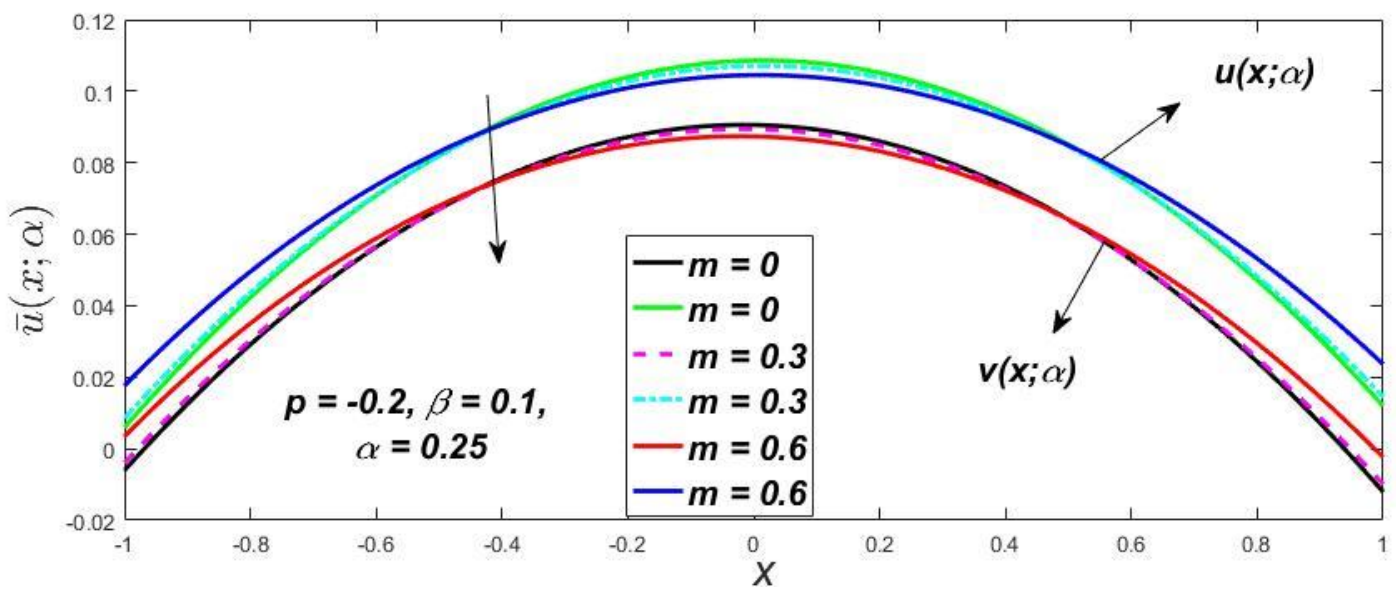

Fig. 20. Fuzzy velocity profiles for influence of $m$.

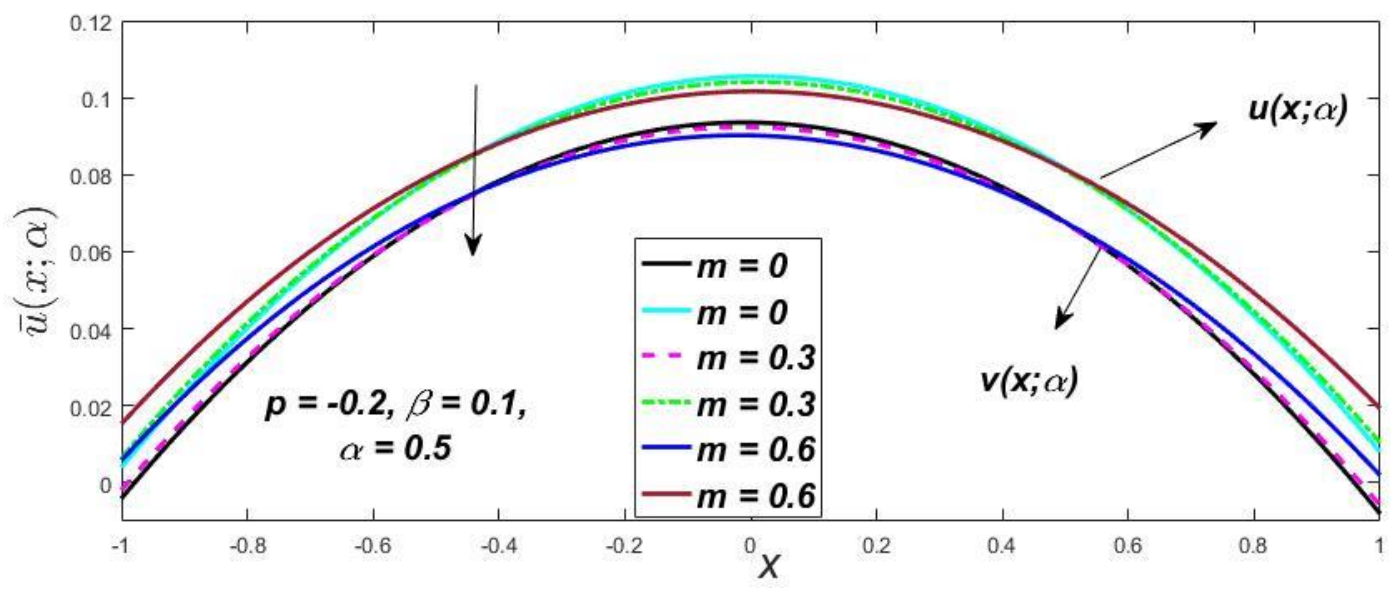

Fig. 21. Fuzzy velocity profiles for influence of $m$.

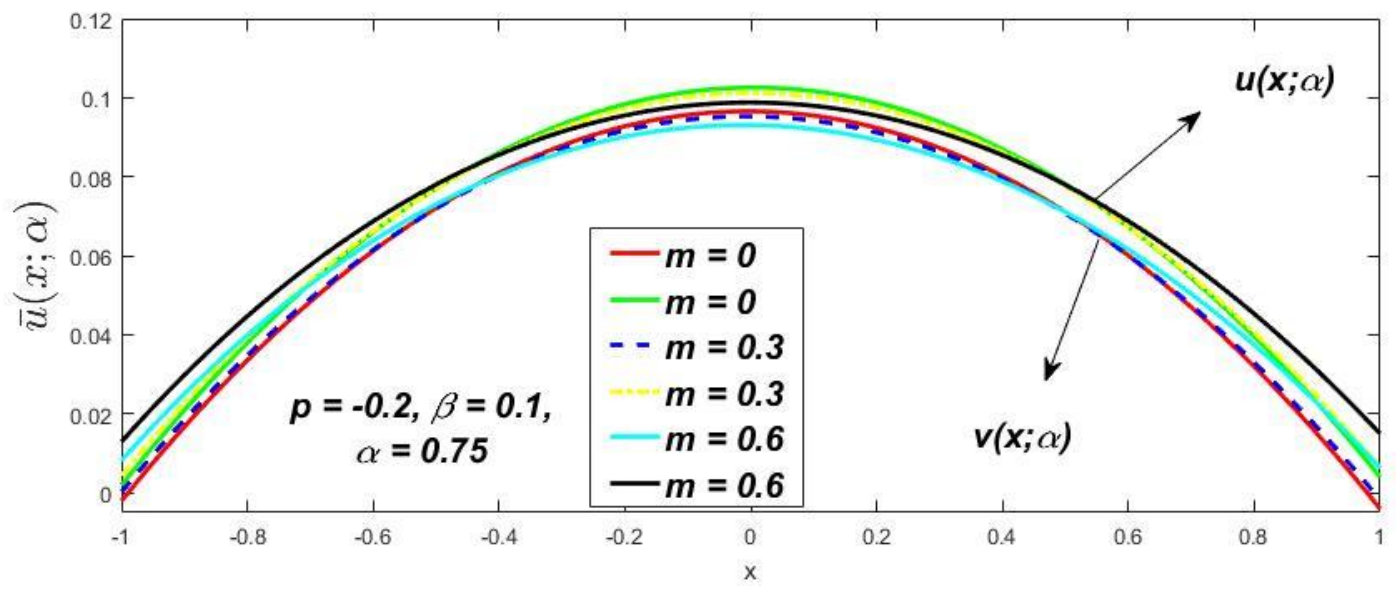

Fig. 22. Fuzzy velocity profiles for influence of $m$. 


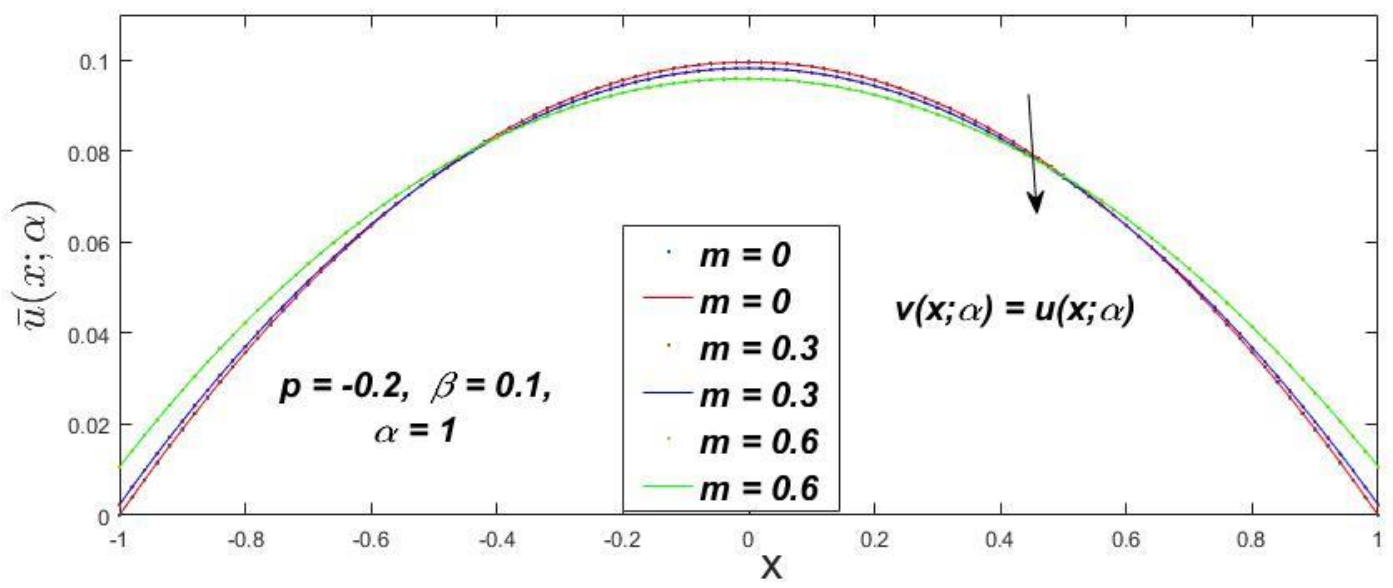

Fig. 23. Fuzzy velocity profiles for influence of $m$.

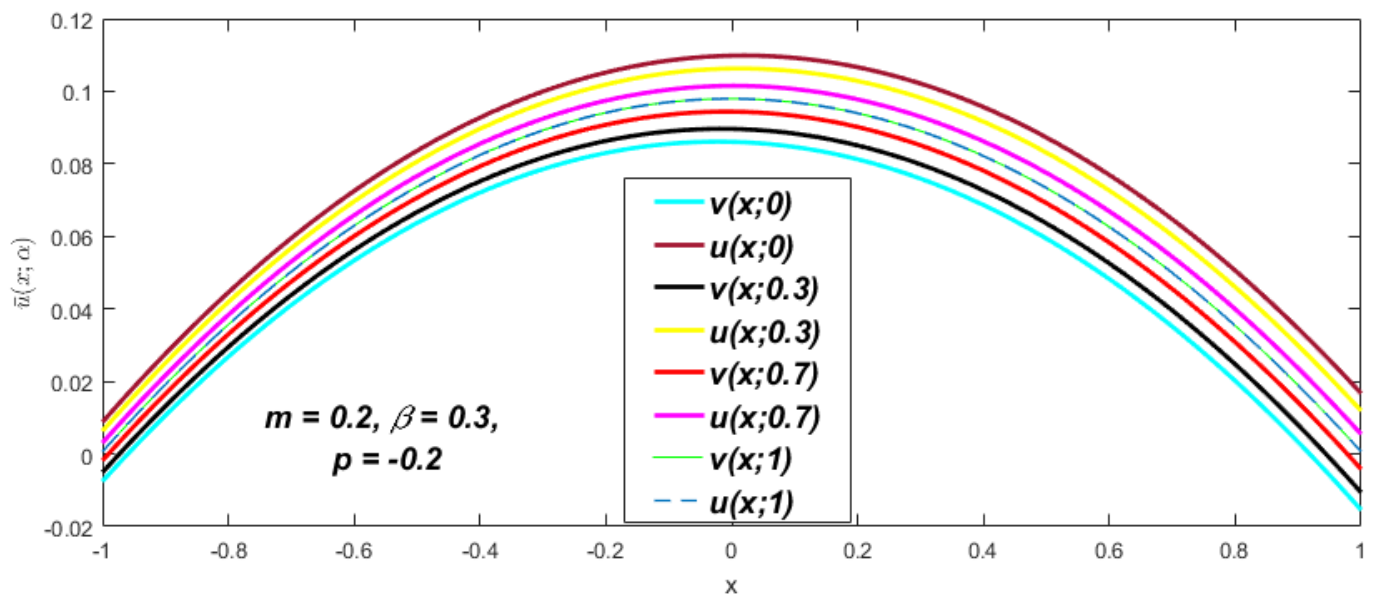

Fig. 24: Lower and upper bounds of velocity profiles for different values of $\alpha$-cut $(0 \leq \alpha \leq 1)$. 


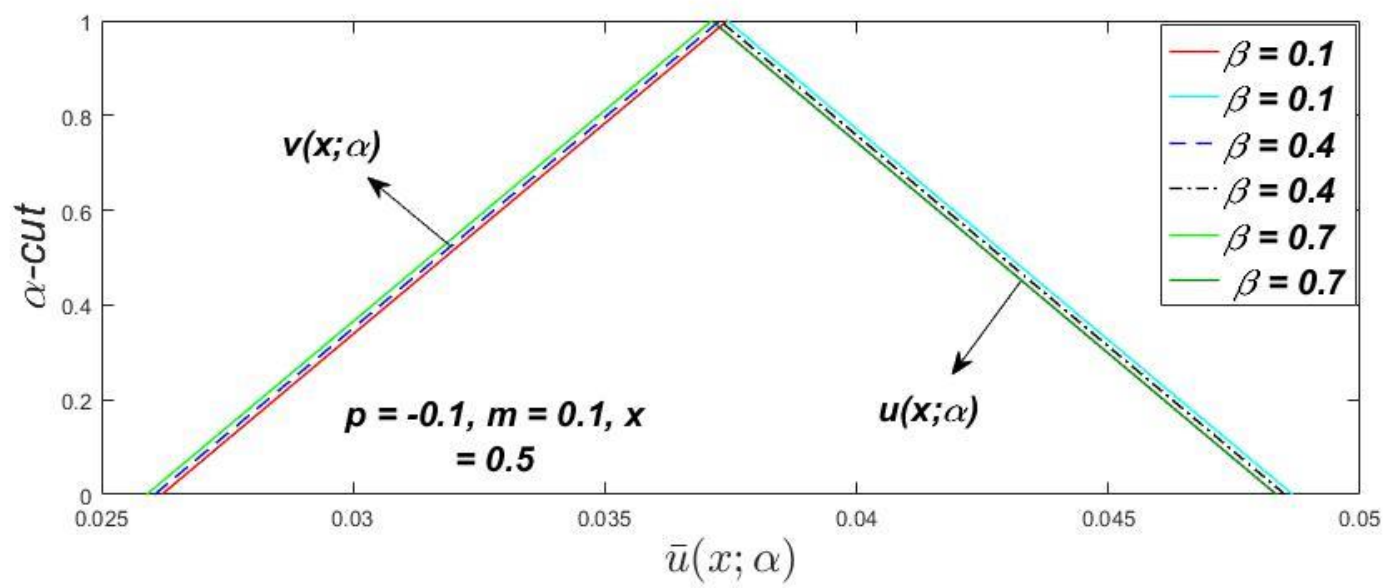

Fig. 25. Triangular membership function of lower and upper bounds of velocity profiles for influence of $\beta$.

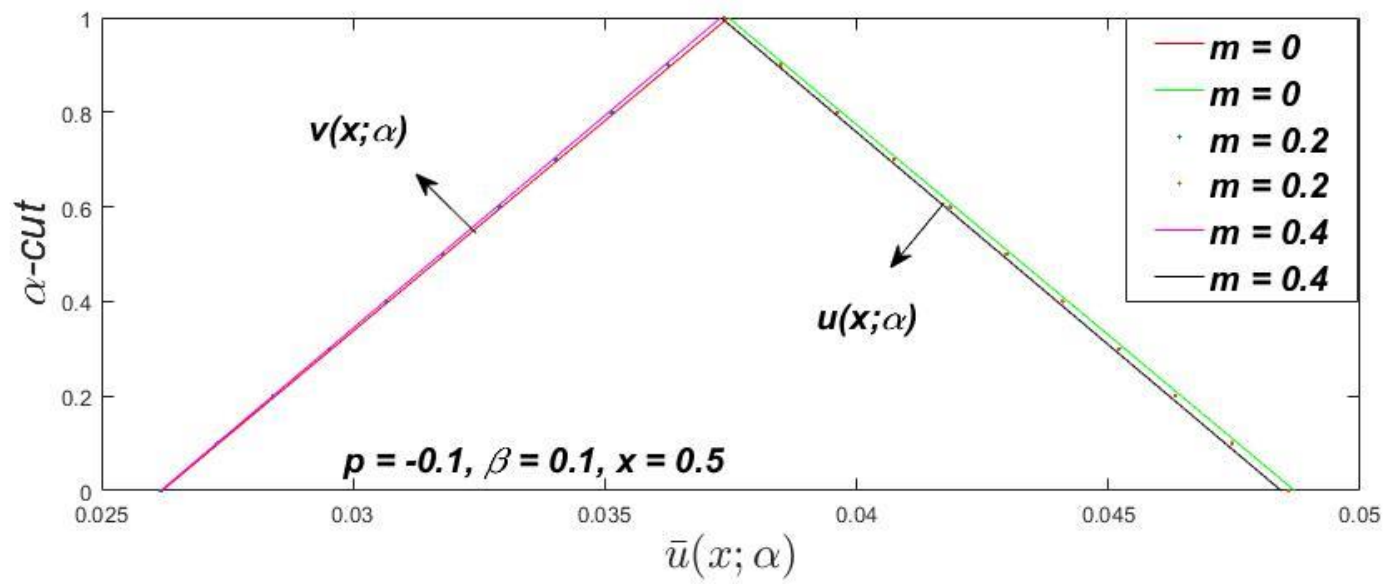

Fig. 26 Triangular membership function of lower and upper bounds of velocity profiles for influence of $m$.

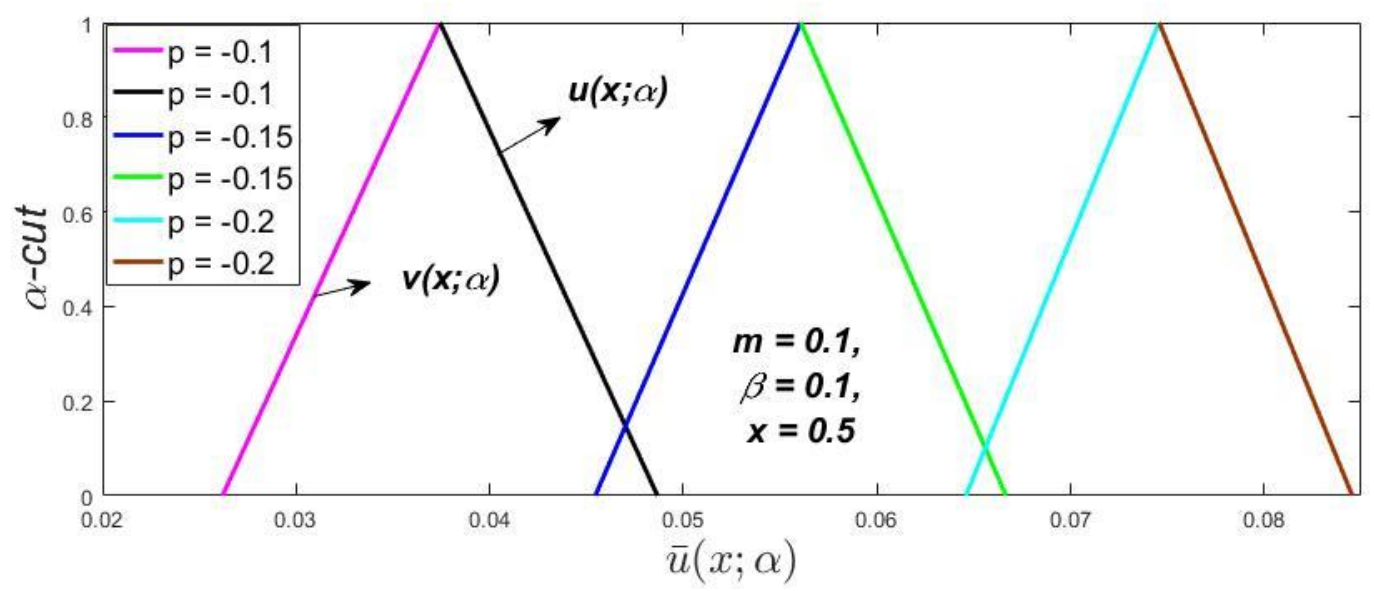

Fig. 27. Triangular membership function of lower and upper bounds of velocity profiles for influence of

$$
p=d p / d y \text {. }
$$


Table 2. Fuzzy solution of lower, mid and upper bounds of velocity profiles at fixed values of $\beta=0.3$, $m=0.2, p=-0.2$ with different values of $\mathrm{x}$.

\begin{tabular}{|c|c|c|c|c|c|c|c|}
\hline$x$ & $\begin{array}{c}v(x ; \alpha) \text { at } \\
\alpha=0\end{array}$ & $\begin{array}{c}\text { Mid value at } \\
\qquad \alpha=0\end{array}$ & $\begin{array}{c}u(x ; \alpha) \text { at } \\
\alpha=0\end{array}$ & $x$ & $\begin{array}{c}v(x ; \alpha) \text { at } \\
\alpha=0\end{array}$ & $\begin{array}{c}\text { Mid value at } \\
\qquad \alpha=0\end{array}$ & $\begin{array}{c}u(x ; \alpha) \text { at } \\
\alpha=0\end{array}$ \\
\hline-1 & -0.00754368 & 0 & 0.00877293 & 0.1 & 0.08606229 & 0.09470363 & 0.10731535 \\
\hline-0.9 & 0.01061348 & 0.02258756 & 0.02762815 & 0.2 & 0.08464196 & 0.09016164 & 0.10212254 \\
\hline-0.8 & 0.02681385 & 0.03875189 & 0.04453579 & 0.3 & 0.08125828 & 0.08413782 & 0.09412950 \\
\hline-0.7 & 0.04106226 & 0.05296998 & 0.06226209 & 0.4 & 0.07591545 & 0.07331703 & 0.08335185 \\
\hline-0.6 & 0.05336013 & 0.06746408 & 0.07941240 & 0.5 & 0.06862011 & 0.06018836 & 0.06981084 \\
\hline-0.5 & 0.06370644 & 0.07915471 & 0.09108383 & 0.6 & 0.05938146 & 0.04714502 & 0.06201222 \\
\hline-0.4 & 0.07209861 & 0.08803667 & 0.09995372 & 0.7 & 0.04821130 & 0.02899539 & 0.04750452 \\
\hline-0.3 & 0.07853329 & 0.09410391 & 0.106015818 & 0.8 & 0.035123944 & 0.01539750 & 0.03455070 \\
\hline-0.2 & 0.08300713 & 0.09700598 & 0.10926482 & 0.9 & 0.020136067 & 0.00815317 & 0.02762828 \\
\hline-0.1 & 0.08551734 & 0.09777557 & 0.10969780 & 1 & -0.00033146 & 0.00000817 & 0.01669182 \\
\hline
\end{tabular}

\section{Generalized Couette Flow}

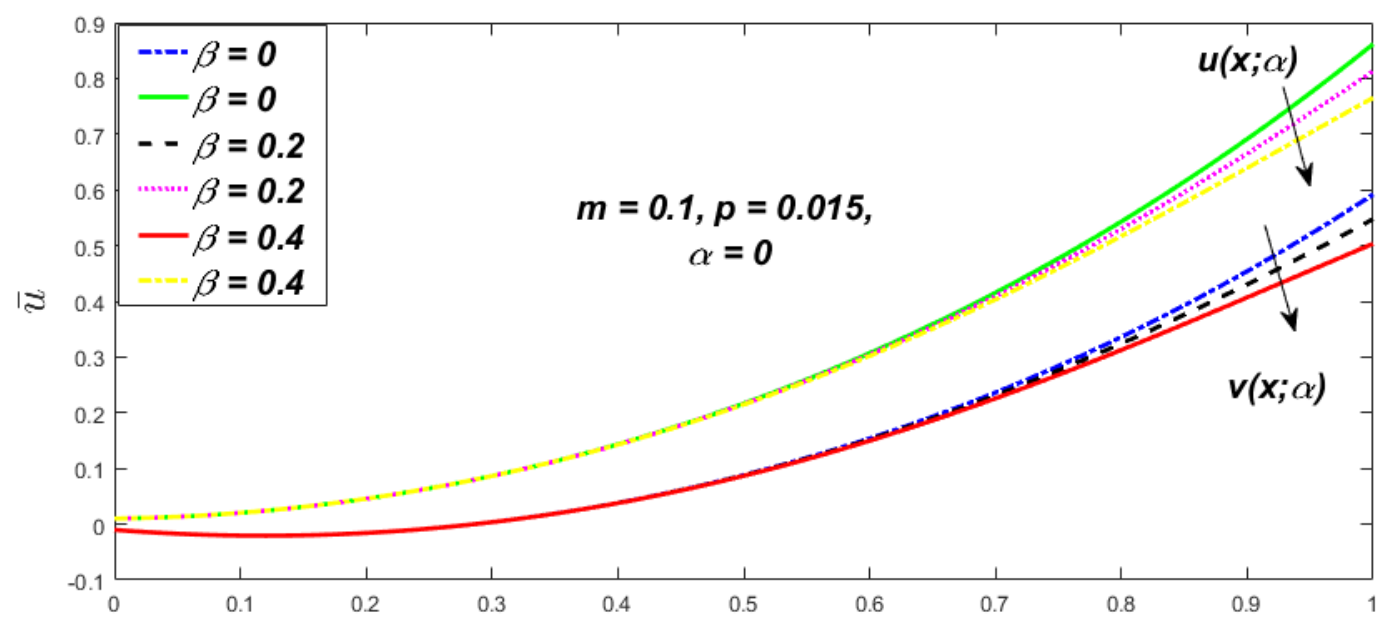

Fig. 28. Fuzzy velocity profiles for influence of $\beta$. 


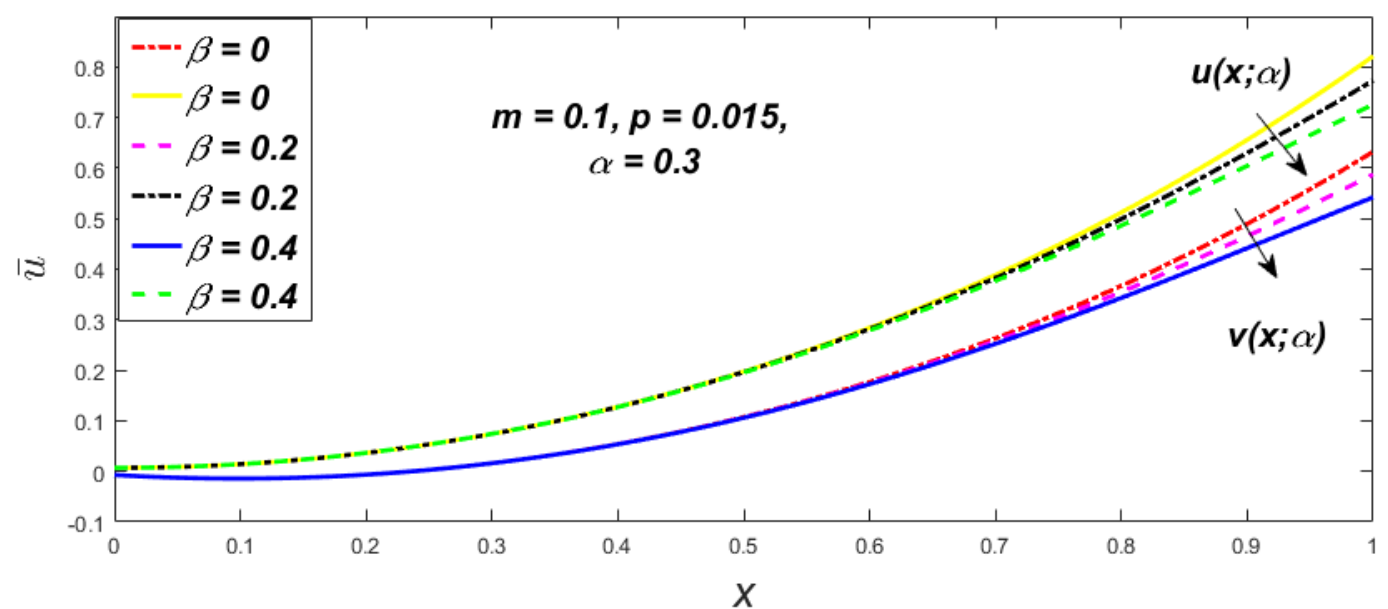

Fig. 29. Fuzzy velocity profiles for influence

of $\beta$.

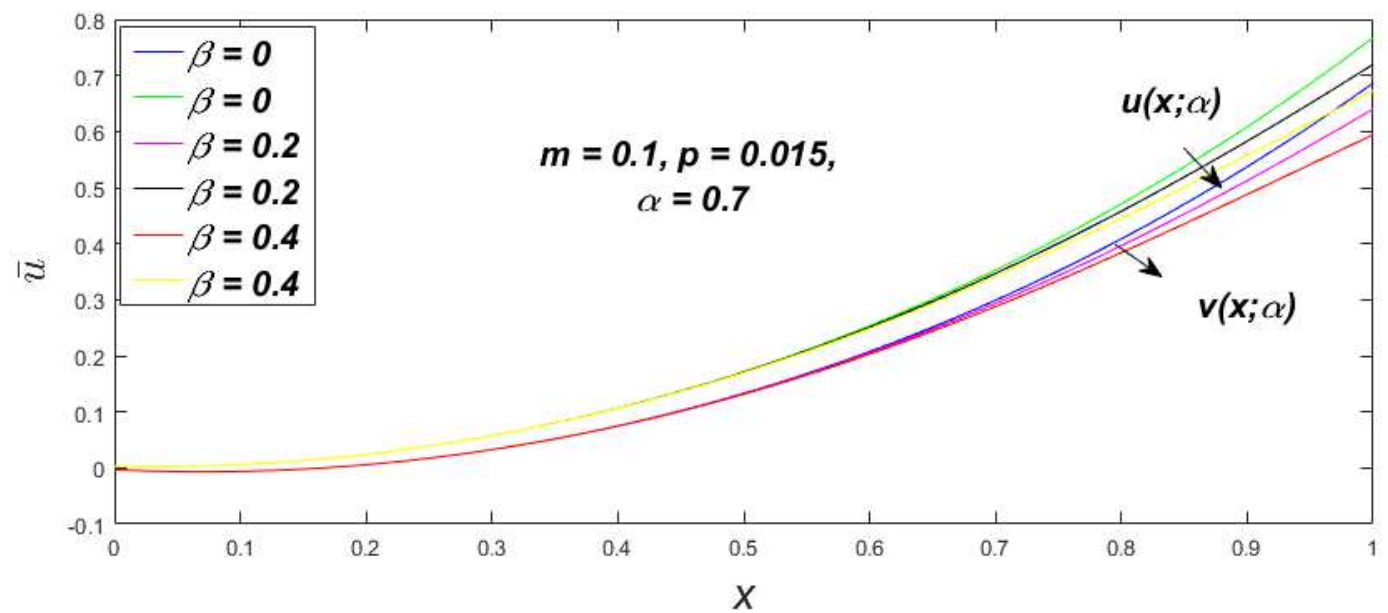

Fig. 30. Fuzzy velocity profiles for influence

of $\beta$.

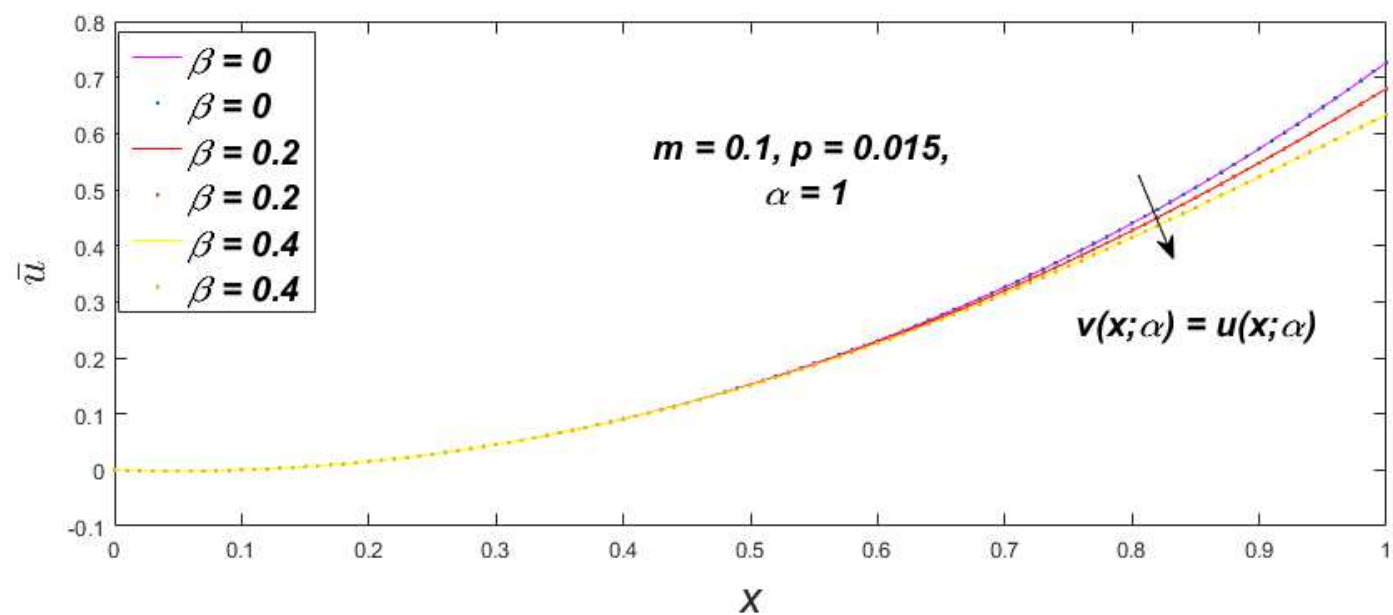


Fig. 31. Fuzzy velocity profiles for influence of $\beta$.

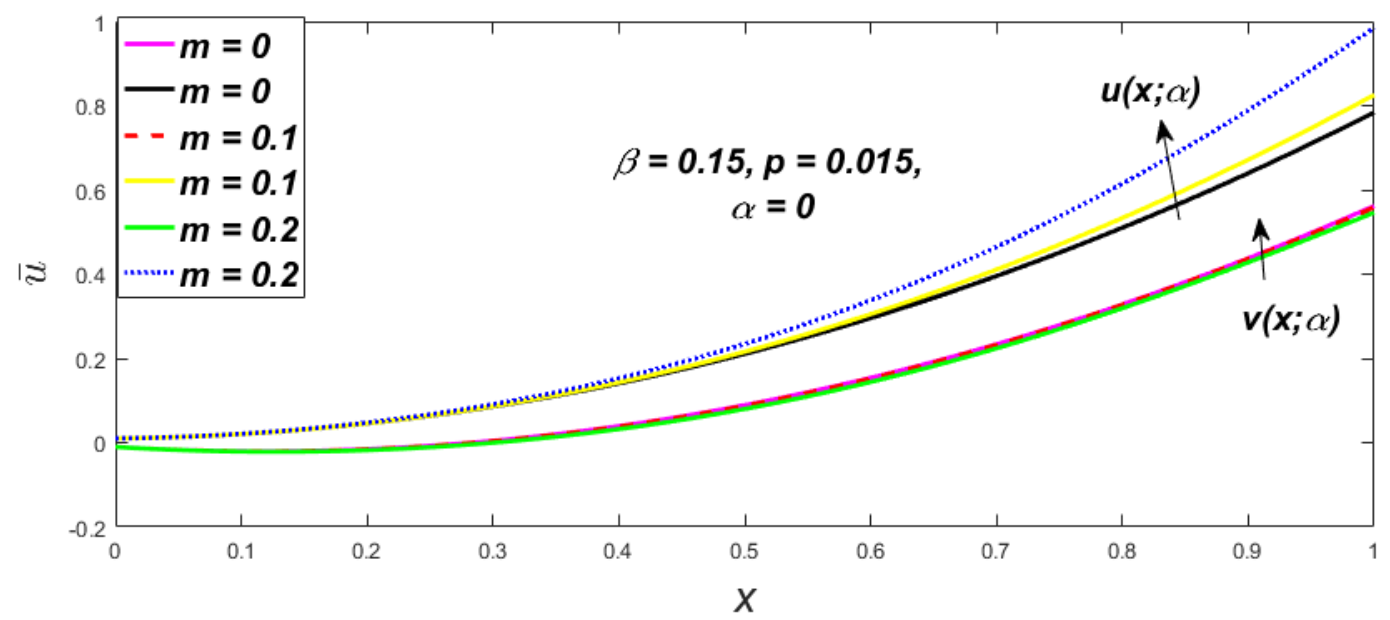

Fig. 32. Fuzzy velocity profiles for influence of $m$.

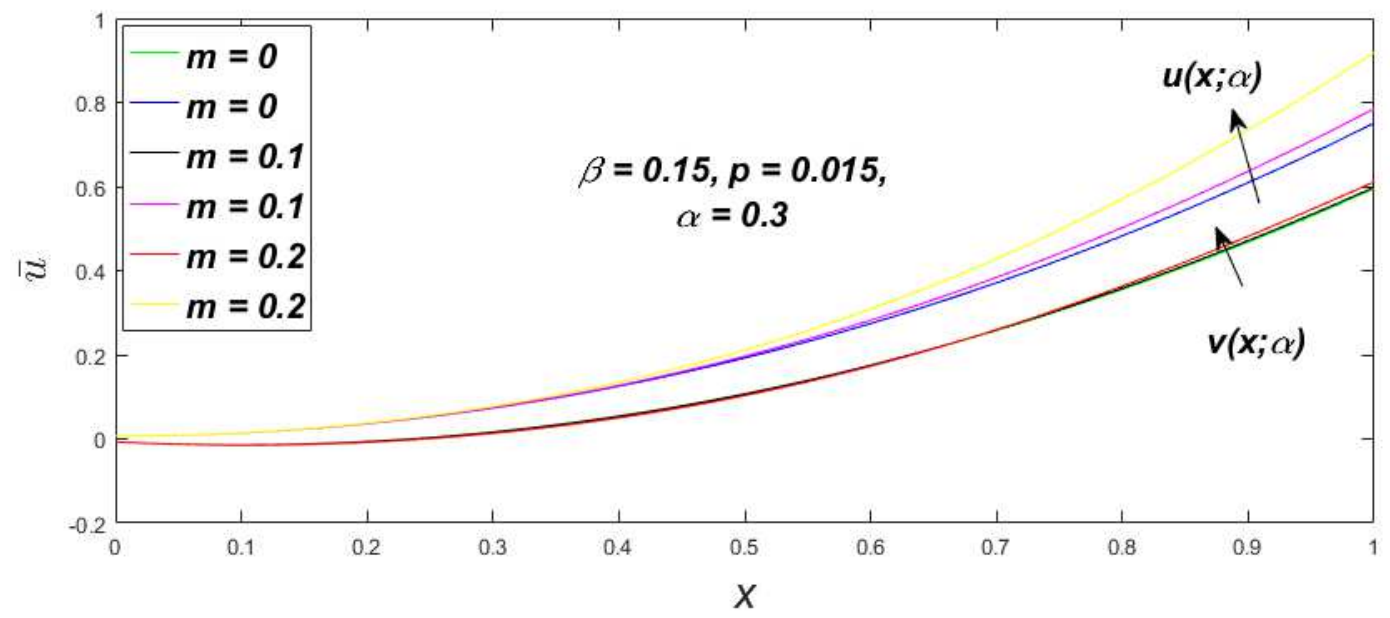


Fig. 33. Fuzzy velocity profiles for influence of

$m$.

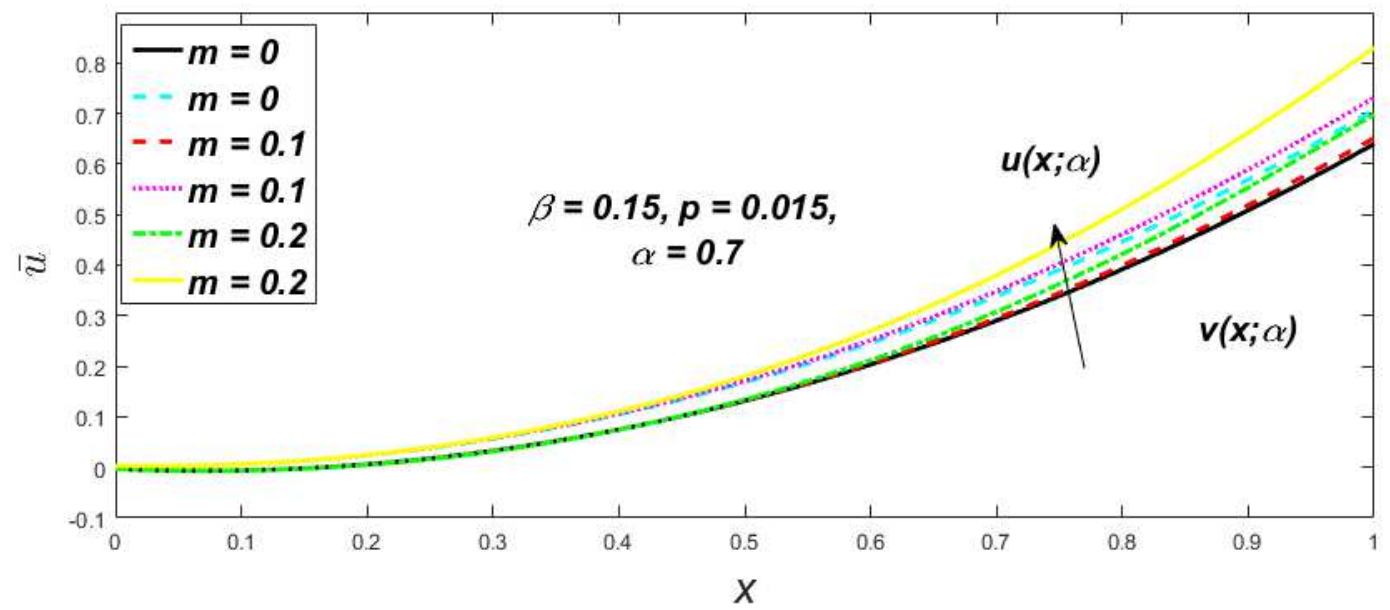

Fig. 34. Fuzzy velocity profiles for influence of

$m$.

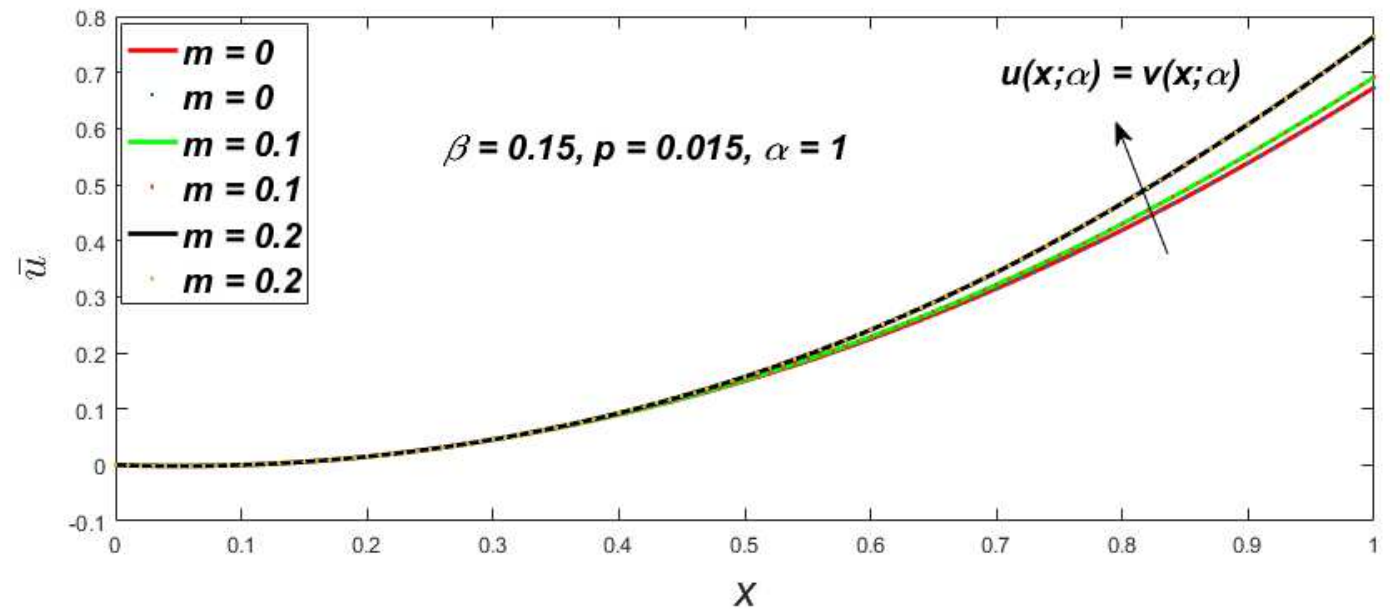

Fig. 35. Fuzzy velocity profiles for influence of 
$m$.

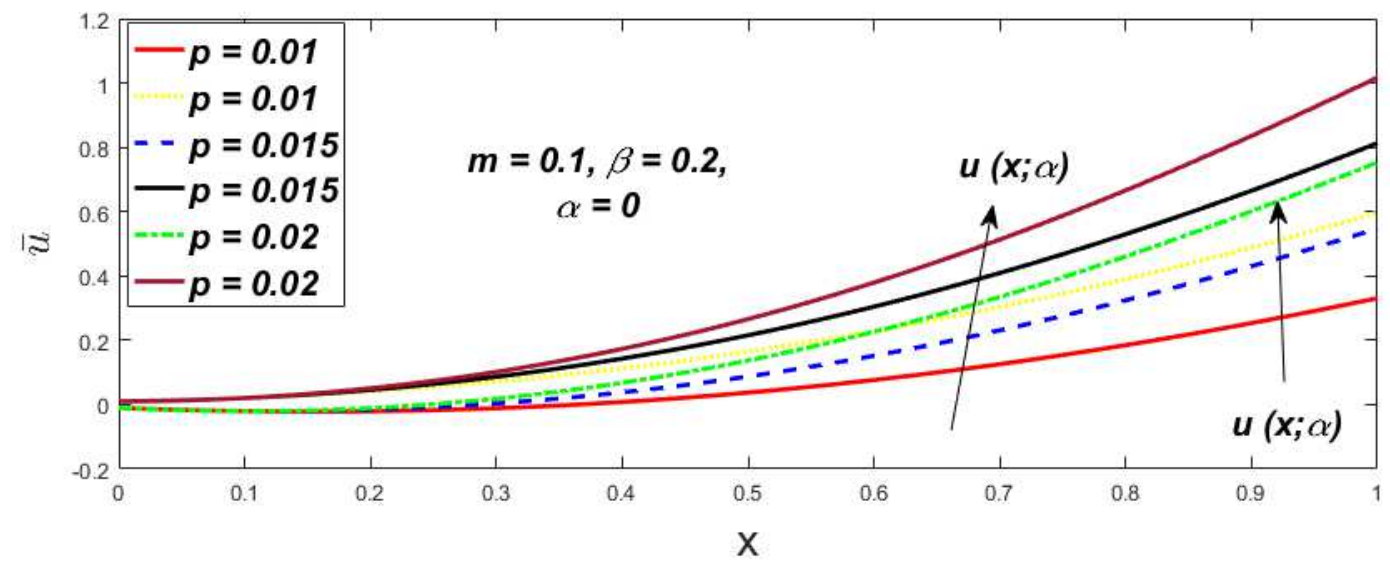

Fig. 36. Fuzzy velocity profiles for influence of $p$.

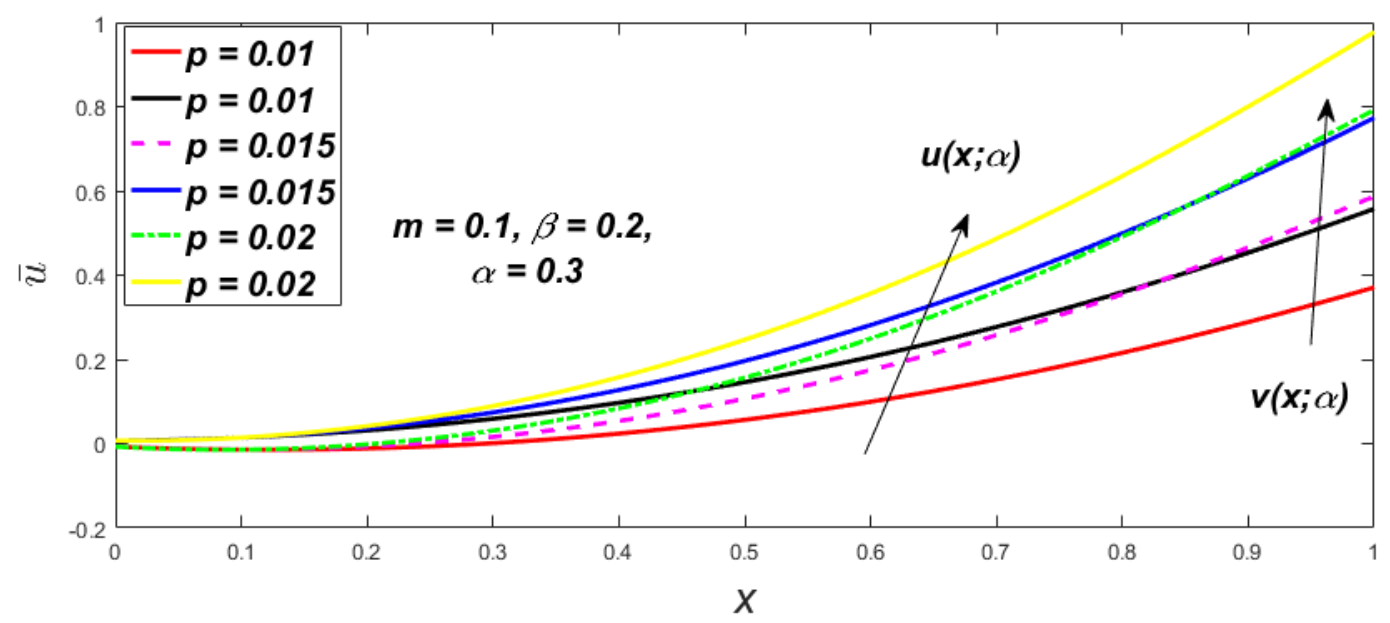

Fig. 37. Fuzzy velocity profiles for influence of $p$. 


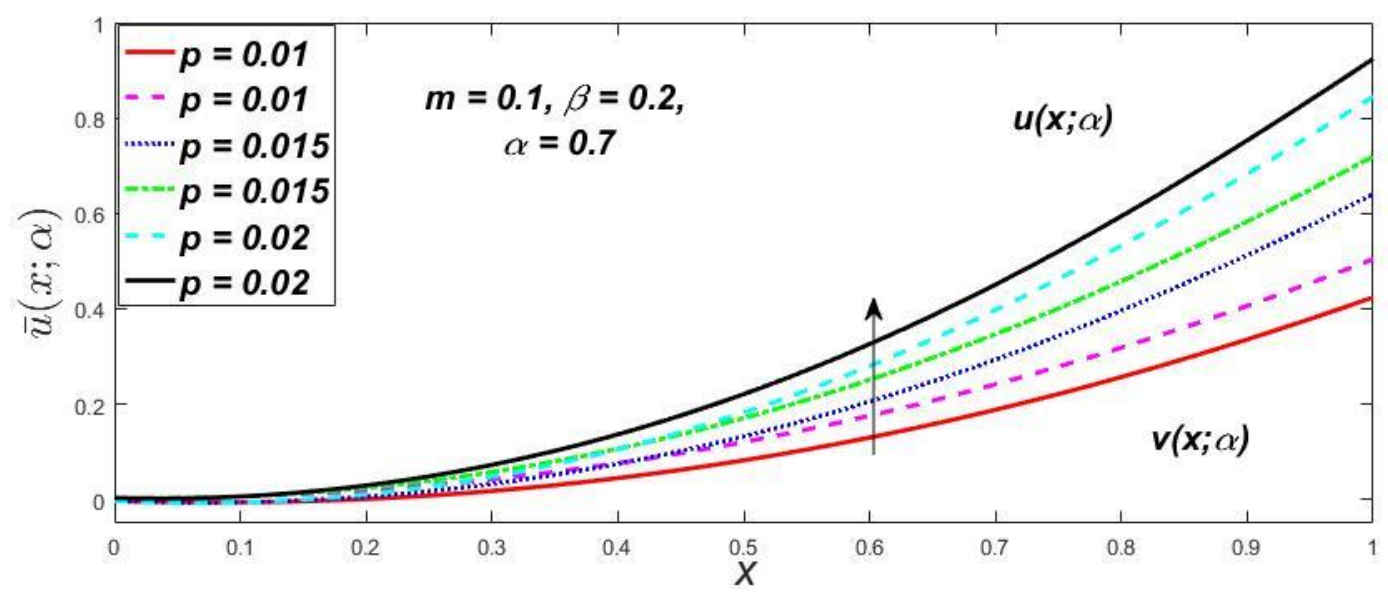

Fig. 38. Fuzzy velocity profiles for influence of $p$.

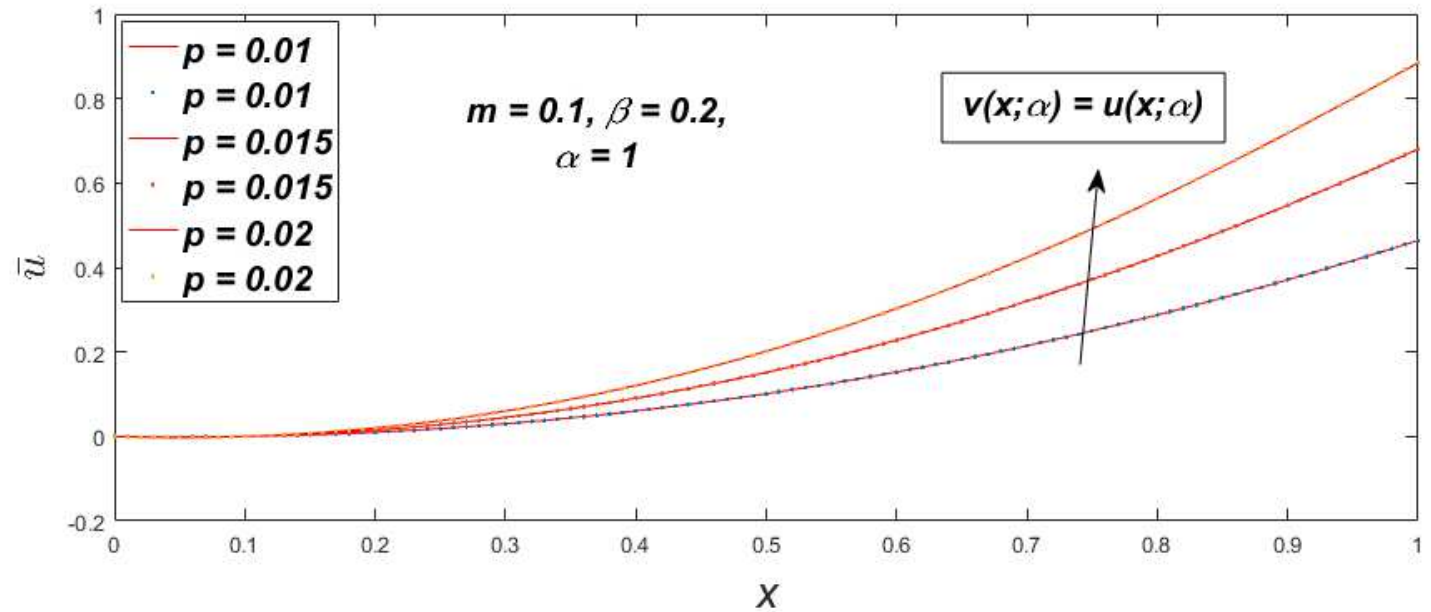

Fig. 39. Fuzzy velocity profiles for influence of $p$. 


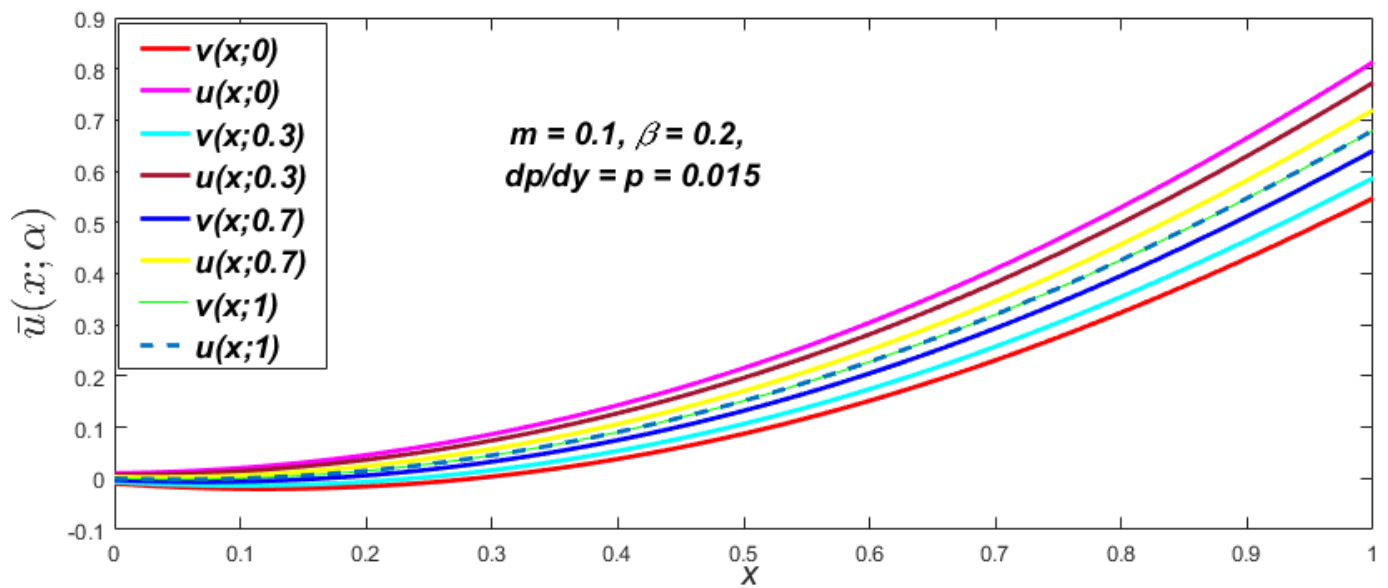

Fig. 40: Lower and upper bounds of velocity profiles for different values of $\alpha$-cut $(0 \leq \alpha \leq 1)$.

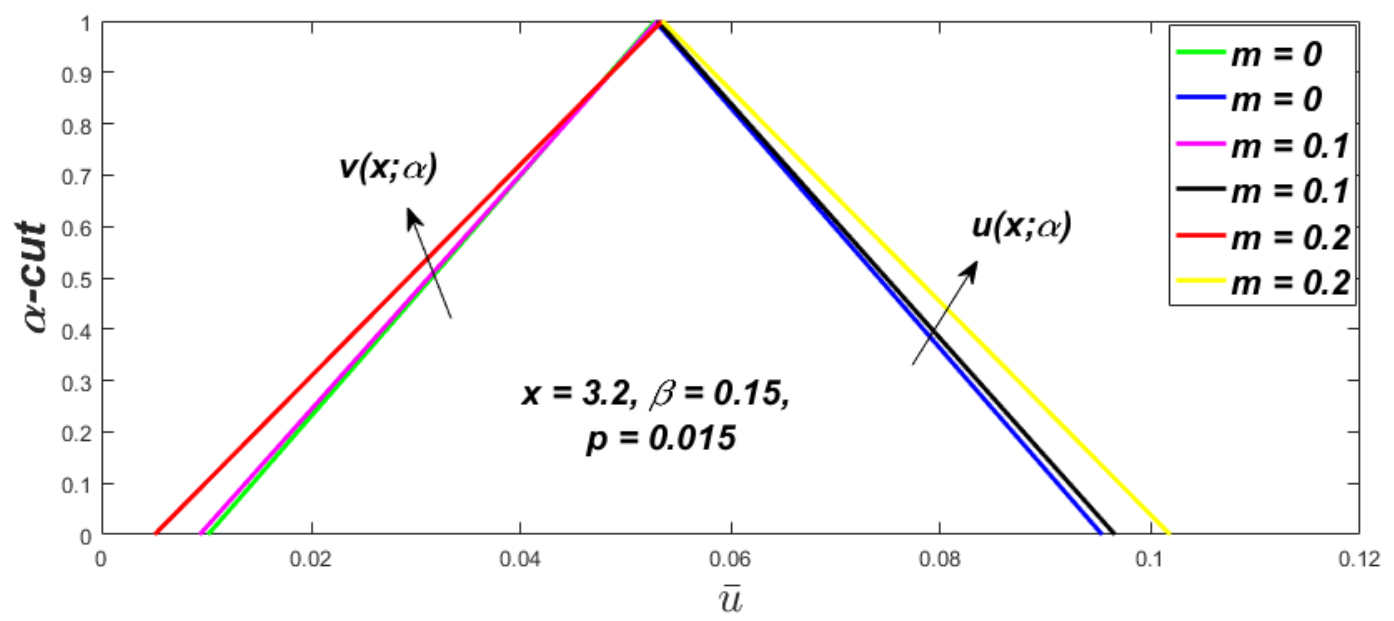

Fig. 41. Triangular membership functions of lower and upper bounds of velocity profiles for influence of $m$. 


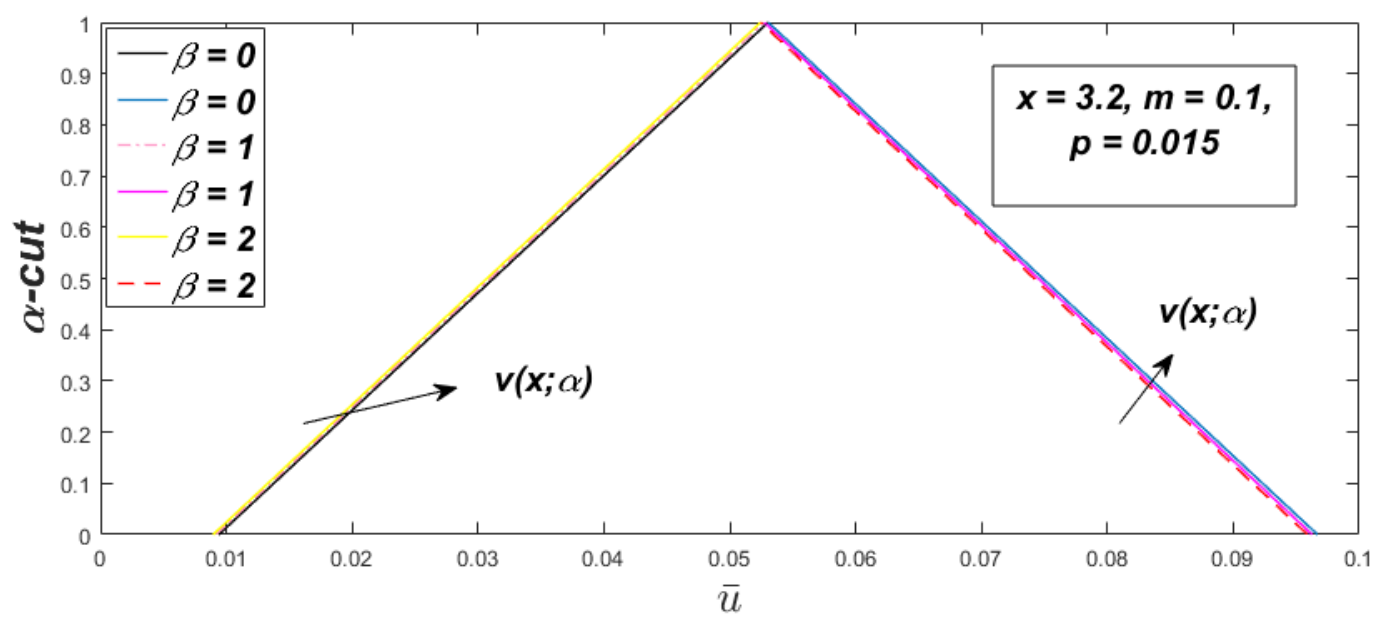

Fig. 42. Triangular membership function of lower and upper bounds of velocity profiles for influence of $\beta$.

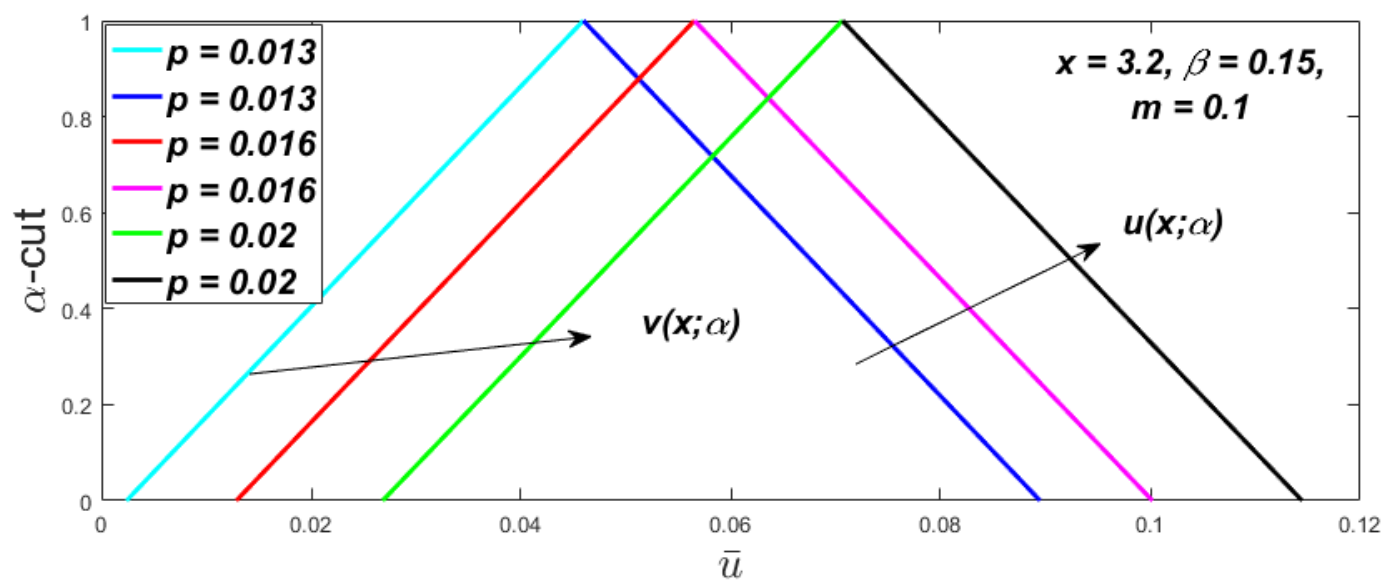

Fig. 43. Triangular membership functions of lower and upper bounds of velocity profiles for influence of $p$.

Table 3. Fuzzy solution of lower, mid and upper bounds of velocity profiles at fixed values of $m=0.01, p=$ $0.015, \beta=0.2$, with different values of $\mathrm{x}$.

\begin{tabular}{|c|c|c|c|}
\hline $\mathrm{x}$ & $v(x ; \alpha)$ at $\alpha=0$ & Mid Value at $\alpha=0$ & $u(x ; \alpha)$ at $\alpha=0$ \\
\hline 0 & -0.010150000141412 & 0 & 0.010149999999511 \\
\hline 0.1 & -0.020300003669159 & -0.000000001466091 & 0.020300000736978 \\
\hline 0.2 & -0.015452766202083 & 0.014996601386919 & 0.045445968975922 \\
\hline 0.3 & 0.004389408523592 & 0.044983248360892 & 0.085577088198192 \\
\hline
\end{tabular}




\begin{tabular}{|c|c|c|c|}
\hline 0.4 & 0.039217240016652 & 0.089946595030480 & 0.140675950044308 \\
\hline 0.5 & 0.089014016780792 & 0.149866061201042 & 0.210718105621291 \\
\hline 0.6 & 0.153754844478778 & 0.224713081851766 & 0.295671319224753 \\
\hline 0.7 & 0.233405596510848 & 0.314450060493039 & 0.395494524475231 \\
\hline 0.8 & 0.327921567007372 & 0.419029024938071 & 0.510136482868770 \\
\hline 0.9 & 0.437245826235755 & 0.538389985488758 & 0.639534144741760 \\
\hline 1 & 0.561307278421598 & 0.672458995535809 & 0.783610712650019 \\
\hline
\end{tabular}

\section{Conclusions}

In this work, we have studied the three fundamental flow problems that frequently arise in the field of fluid dynamics, namely, plane Couette flow, plane Poiseuille flow and generalized Couette flow of a non-Newtonian fluid under the influence of MHD in a fuzzy environment. The dimensionless non-linear governing equations converted into FDEs with fuzzified boundary conditions and find their solutions using ADM. For plane Couette flow, plane Poiseuille flow and generalized Couette flows, the triangular fuzzy numbers are used for uncertainty on the dynamic behavior of lower and upper velocity profiles. In these three flow problems, velocity profiles (lower and upper) increases with increasing the non-Newtonian fluid parameter $\beta$ and fuzzy parameter $\alpha \in[0,1])$ while the velocity profiles decreasing with increasing magnetic parameter $m$. Results indicated that the range of calculated lower and upper velocity profiles depend upon fuzzy parameter $\alpha$. The less width of fuzzy velocity profiles so uncertainty is less than the boundary is less sensitive. While, width of fuzzy velocity profiles are greater so uncertainty is maximum then the boundary is more sensitive. The obtained results are an envelope with a crisp solution between the upper and lower solutions. So, fuzzy velocity profiles are the generalization of the crisp velocity profile of third grade fluid between two parallel plates. 


\section{Authors' contributions}

All authors have equal contributions on the paper. All authors have read and approved the final version of the paper.

\section{Funding}

There is no funding

Compliance with ethical standards

Conflict of interest

The authors declare that they have no conflict of interest

\section{Ethical approval}

This article does not contain any studies with human participants \& animals performed by any of the authors.

\section{References}

[1] C. Truesdell, W. Noll, The non-linear field's theories of mechanics. Third ed. Springer, 2004.

[2] K. R. Rajagopal, On the stability of third grade fluids Arch Mech. 32(6), 867-875, 1980.

[3] K. R. Rajagopal, Thermodynamics and stability of fluids of third grade. Pros R Scotland A, 339, 351-377, 1980.

[4] A. M. Siddiqui, R. Mahmood, Q. K. Ghori, Homotopy perturbation method for thin film flow of a third-grade fluid down an inclined plane. Chaos Solitons and Fractals, 35, 140-147, 2008.

[5] T. Hayat, R. Ellahi, F. M. Mahomed, Exact solutions for thin film flow of a third-grade fluid down an inclined plane. Chaos, Solitons and Fractals, 38, 1336-1341, 2008.

[6] M. Sajid, T. Hayat, The application of Homotopy analysis method to thin film flows of a third order fluid, Chaos, Solitons and Fractals, 38, 506-515, 2008.

[7] R. A. Shah, S. Islam, M. Zeb, I. Ali, Optimal homotopy asymptotic method for thin film flows of a third order fluid, J. of Adv. Research in Scientific Computing, 2, 1-14, 2011. 
[8] A.M. Siddiqui, A. A. Farooq, T. Haroon, M. A. Rana, B. S. Babcock, Application of He's Variational Iterarion method for solving thin film flow problem arising in Non-Newtonian fluid mechanics, World Journal of Mechanics, 2, 138-142, 2012.

[9] S. Iqbal, K. M. Abualnaja, Galerkin's finite element formulation for thin film flow of a third order fluid down an inclined plane, Science International (Lahore), 26 (4), 1403-1405, 2014.

[10] S. I. Natarov, C. P. Conrad, The role of Poiseuille flow in creating depth-variation of asthenospheric shear, Geophys. J. Int., 190 (3), 1297-1310, 2012.

[11] T. Hayat, M. Khan, M. Ayub, Couette and Poiseuille flows of an Oldroyd 6-constant fluid with magnetic field, J. Math. Anal. Appl. 298 (1), 225-244, 2004.

[12] T. Hayat, R. Naz, M. Sajid, On the homotopy solution for Poiseuille flow of a fourth grade fluid, Commun. Nonlinear Sci. Numer. Simul. 15 (3), 581-589, 2010.

[13] T. Chinyoka, O. D. Makinde, Analysis of transient generalized Couette flow of a reactive variable viscosity third-grade liquid with asymmetric convective cooling, Math. Comput. Model, 54 (1), 160-174, 2011.

[14] M. Khan, C. Fetecau, T. Hayat, MHD transient flows in a channel of rectangular crosssection with porous medium. Physics letters A, 369 (1), 44-54, 2007.

[15] T. Hayat, T. Haroon, S. Asghar, , A. M. Siddiqui, MHD flow of a third-grade fluid due to eccentric rotations of a porous disk and a fluid at infinity. International journal of non-linear mechanics, 38 (4), 501-511, 2003.

[16] T. Hayat, M. Khan, M. Ayub, Couette and Poiseuille flows of an Oldroyd 6-constant fluid with magnetic field. J. of mathematical analysis and applications, 298 (1), 225-244, 2004.

[17] T. Hayat, K. Hutter, S. Asghar, A. M. Siddiqui, MHD flows of an Oldroyd-B fluid. Mathematical and Computer Modelling, 36 (9-10), 987-995, 2002.

[18] S. Islam, Homotopy perturbations analysis of couette and poiseuille flows of a third-grade fluid with magnetic field. Science International, 22 (3), 2010.

[19] M. Kamran, I. Siddique, MHD Couette and Poiseuille Flow of a Third Grade Fluid, Open J. Math. Anal., 1 (2), 01-19, 2017.

[20]G. Adomian, A review of the decomposition method and some recent results for nonlinear equations. Math Comput Model, 13 (7), 287-99, 1992.

[21] G. Adomian, Solving frontier problems of physics: the decomposition method. Dordrecht: Kluwer; 1994. 
[22] Y, Cherruault, G. Adomian, Decomposition method: a new proof of convergence. Math Comput. Model, 18 (12), 103-6, 1993.

[23] A. M. Siddiqui, M. Hameed, B. M. Siddiqui, Q. K. Ghori, Use of Adomian decomposition method in the study of parallel plate flow of a third grade fluid, commu-nications in Nonlinear Science and Numerical Simulation, 15 (9), 2388-2399, 2010.

[24] U. M. Pirzada, D. C. Vakaskar, Solution of fuzzy heat equations using adomian decomposition method, Int. J. Adv. Appl. Math. Mech., 3 (1), 87-91, 2015.

[25] M. Paripour, E. Hajilou, H. Heidari, Application of Adomian decomposition method to solve hybrid fuzzy differential equations, J. of Taibah Uni. for Sci., 57, 1658-3655, 2014.

[26] A. M. Siddiqui, T. Haroon, S. Bhatti, A. R. Ansari, A comparison of the adomian and homotopy perturbation methods in solving the problem of squeezing flow between two circular plates. Mathematical Modelling and Analysis, 15 (4), 491-504, 2010.

[27] U. Biswal, S. Chakraverty, B.K. Ojha, Natural convection of nanofluid flow between two vertical flat plates with imprecise parameter, Coupled Systems Mechanics, 9(3), 219-235, 2020.

[28] L. A. Zadeh, Fuzzy Sets. Inf. Control 8 (3), 338-353, 1965.

[29] D. Dubois, H. Prade, Operations on fuzzy numbers, International J. of Systems Science, 9 (6), 613-626, 1978.

[30] S. Seikala, On the fuzzy initial value problem, Fuzzy Sets and Systems, 24(3), 319-330, 1987.

[31] O. Kaleva, Fuzzy differential equations, Fuzzy Sets and Systems, 24 (3), 301-317, 1987.

[32] A. Kandel, W. J. Byatt, Fuzzy differential equations, in: Proceedings of International Conference Cybernetics and Society, Tokyo, 1213- 1216, 1978.

[33] J. J. Buckley, T. Feuring, Y. Hayashi, Linear systems of first order ordinary differential equations: fuzzy initial conditions, Soft Comput. 6, 415-421, 2002.

[34] J. J. Nieto, The Cauchy problem for continuous fuzzy differential equations, Fuzzy Sets and Systems, 102, 259-262, 1999.

[35] V. Lakshmikantham, R. N. Mohapatra, Basic properties of solutions of fuzzy differential equations, Nonlinear Studies, 8, 113 -124, 2000. 
[36] S. Salahshour, A. Ahmadian, A. Mahata, The Behavior of Logistic Equation with Alley Effect in Fuzzy Environment: Fuzzy Differential Equation Approach. Int. J. Appl. Comput. Math, 4, 62, 2018.

[37] M. S. Hashemi, J. Malekinagad, H. R. Marasi, Series solution of the system of fuzzy differential equations, Adv. in Fuzzy Systems, 16, 2012.

[38] C. Tzimopoulos, G. Papaevangelou, K. Papadopoulos, C. Evangelides, G. Arampatzis, Fuzzy Analytical Solution to Vertical Infiltration. Journal of Software Engineering and Applications, 13, 41-66, 2020.

[39] N. Gasilov, S .E. Amrahov, A.G. Fatullayev, A geometric approach to solve fuzzy linear systems of differential equations, Appl. Math. Inf. Sci., 5 (3), 484-499, 2011.

[40] A. Khastan, J.J. Nieto, A boundary value problem for second order fuzzy differential equations, Nonlinear Anal., 72, 3583-3593, 2010.

[41] S. P. Mondal, T. K. Roy, First Order Linear Homogeneous Ordinary Differential Equation in Fuzzy Environment Based On Laplace Transform, J. of Fuzzy Set Valued Anal., 1-18, 2013.

[42] H. Zarei, A. V. Kamyad, A. A. Heydari, Fuzzy Modeling and Control of HIV Infection, Computational and Mathematical Methods in Medicine, Article ID 893474, 17, 2012.

[43] S. P. Mondal, S. Banerjee, T. K. Roy, First Order Linear Homogeneous Ordinary Differential Equation in Fuzzy Environment, Int. J. Pure Appl. Sci. Technol, 14 (1), 16-26, 2013.

[44] M. Guo, X. Xue, R. Li, Impulsive functional differential inclusions and fuzzy population models, Fuzzy Sets and Systems, 138, 601-615, 2003.

[45] L. C. Barros, R. C. Bassanezi, P. A. Tonelli, Fuzzy modelling in population dynamics, Ecol. Model, 128, 27-33, 2000.

[46] M. Z. Ahmad, B. De Baets, A Predator-Prey Model with Fuzzy Initial Populations, IFSAEUSFLAT, 2009.

[47] J. Casasnovas, F. Rossell, Averaging fuzzy bio-polymers, Fuzzy Sets and Systems, 152, 139$158,2005$.

[48] G. L. Diniz, J. F. R. Fernandes, J. F. C. A. Meyer, L. C. Barros, A fuzzy Cauchy problem modeling the decay of the biochemical oxygen demand in water, Proceedings Joint 9th IFSA World Congress and 20th NAFIPS International Conference, 1, 512-516, IEEE, 2001. 
[49] A. Bencsik, B. Bede, J. Tar, J. Fodor, Fuzzy differential equations in modeling hydraulic differential servo cylinders, In: Third Romanian-Hungarian joint symposium on applied computational intelligence (SACI), Timisoara, Romania, 2006.

[50] B. Bede, I. J. Rudas, J. Fodor, Friction Model by Fuzzy Differential Equations, IFSA, LNAI 4529, 23-32, Springer-Verlag Berlin Heidelberg, 2007.

[51] M. S. El Naschie, From experimental quantum optics to quantum gravity via a fuzzy Khler manifold, Chaos, Solitons and Fractals, 25, 969-977, 2005.

[52] T. Allahviranloo, S. Salahshour, Applications of fuzzy Laplace transforms, Soft Computing, 17 (1), 145-158, 2013.

[53] M. Oberguggenberger, S. Pittschmann, Differential equations with fuzzy parameters, Math. Mod. Syst., 5, 181-202, 1999.

[54] S. Hajighasemi, T. Allahviranloo, M. Khezerloo, M. Khorasany, S. Salahshour, Existence and uniqueness of solutions of fuzzy Volterra integro-differential equations, Information Processing and Management of Uncertainty in Knowledge-Based, 81, 491-500, 2010.

[55] A. El Allaoui, S. Melliani, and L. S. Chadli, A mathematical fuzzy model to giving up smoking, IEEE $6^{\text {th }}$ International Conference on Optimization and Applications (ICOA), 1-6, 2020.

[56] H. C. Bhandari, K. Jha, An Analysis of Microbial Population of Chemo-stat Model in fuzzy environment, The Nepali Math. Sc. Report, 36(1), 2, 2019.

[57] A. Rajkumar, C. Jesuraj, Mathematical model for dengue virus infected populations with fuzzy differential equations Advanced Informatics for Computing Research, Communications in Computer and Information Science, Vol. 955, Singapore: Springer, 206-217, 2018.

[58] P. B. Dhandapani, D. Baleanu, J. Thippan, V. Sivakumar, On stiff, fuzzy IRD-14 day average transmission model COVID-19 pandemic disease, AIMS Bioengineering, 7(4), 208-223, 2020.

[59] U. Biswal, S. Chakraverty, B.K. Ojha, Natural convection of nanofluid flow between two vertical flat plates with imprecise parameter, Coupled Systems Mechanics, 9(3), 219-235, 2020.

\section{Appendix}




$$
\begin{aligned}
& A_{1}=\frac{11 \alpha}{200}, A_{2}=\frac{17 \alpha-6}{200}, A_{3}=\frac{107 \alpha-30}{200}, A_{4}=\frac{\alpha-1}{100}, \\
& \mathrm{~B}_{1}=\frac{41-21 \alpha}{400}, \mathrm{~B}_{2}=\frac{53-33 \alpha}{400}, \mathrm{~B}_{3}=\frac{347-207 \alpha}{400}, \mathrm{~B}_{4}=\frac{1-\alpha}{100}, \\
& D=\frac{(p+1)(\alpha-1)-50 p}{100}, \mathrm{E}=\frac{(p+1)(\alpha-1)}{100}, \\
& K=\frac{\beta D^{5}}{5 p}+\frac{\beta D^{3}\left(D^{4}-(p+D)^{4}\right)}{4 p^{2}}+\frac{\beta D^{6}}{5 p}+\frac{m^{2} D^{3}(p+4 D+12 E)}{72 p}, \\
& R=\frac{\beta(p+D)^{6}}{60 p^{3}}+\frac{\beta\left(D^{4}-(p+D)^{4}\right)}{12 p}-\frac{D^{4}}{4 p}-\frac{\beta D^{6}}{60 p^{3}}-\mathrm{m}^{2}\left(\frac{6 D+p+30 E-5 p-20 D-60 E}{720}\right), \\
& L=\frac{\beta(p+D)^{5}}{5 p}+\frac{\beta(p+D)^{6}}{5 p}+\frac{3 \beta(p+D)^{3}\left(D^{4}-(p+D)^{4}\right)}{4 p^{2}}-\frac{\beta D^{5}}{5 p}-\frac{\beta D^{3}\left(D^{4}-(p+D)^{4}\right)}{4 p^{2}} \\
& -\frac{\beta D^{6}}{5 p}-m^{2}\left[\begin{array}{l}
\frac{p^{2}(2 p+3)}{180}+\frac{D p^{2}}{6}+\frac{7 D^{2} p}{24}-\frac{4 E D p+D^{3}}{6}+\frac{(p+4 D+12 E)(p+D)^{3}}{72 p} \\
+\frac{E p^{2}}{4}-\frac{D^{3}(p+4 D+12 E)}{72 p}-E D^{2}
\end{array}\right], \\
& D_{1}=\frac{(p+1)(1-\alpha)-50 p}{100}, \mathrm{E}_{1}=\frac{(p+1)(1-\alpha)}{100}, \\
& K_{1}=\frac{\beta D_{1}^{5}}{5 p}+\frac{\beta D_{1}^{3}\left(D_{1}^{4}-\left(p+D_{1}\right)^{4}\right)}{4 p^{2}}+\frac{\beta D_{1}^{6}}{5 p}+\frac{m^{2} D_{1}^{3}\left(p+4 D_{1}+12 E_{1}\right)}{72 p}, \\
& R_{1}=\frac{\beta\left(p+D_{1}\right)^{6}}{60 p^{3}}+\frac{\beta\left(D_{1}^{4}-\left(p+D_{1}\right)^{4}\right)}{12 p}-\frac{D_{1}^{4}}{4 p}-\frac{\beta D_{1}^{6}}{60 p^{3}}-\mathrm{m}^{2}\left(\frac{6 D_{1}+p+30 E_{1}-5 p-20 D_{1}-60 E_{1}}{720}\right), \\
& L_{1}=\frac{\beta\left(p+D_{1}\right)^{5}}{5 p}+\frac{\beta\left(p+D_{1}\right)^{6}}{5 p}+\frac{3 \beta\left(p+D_{1}\right)^{3}\left(D_{1}^{4}-\left(p+D_{1}\right)^{4}\right)}{4 p^{2}}-\frac{\beta D_{1}^{5}}{5 p}-\frac{\beta D_{1}^{3}\left(D_{1}^{4}-\left(p+D_{1}\right)^{4}\right)}{4 p^{2}} \\
& -\frac{\beta D_{1}^{6}}{5 p}+m^{2}\left[\begin{array}{l}
-\frac{p^{2}(2 p+3)}{180}-\frac{D_{1} p^{2}}{6}-\frac{7 D_{1}^{2} p}{24}+\frac{4 E_{1} D_{1} p+D_{1}^{3}}{6}+E_{1} D_{1}^{2} \\
-\frac{\left(p+4 D_{1}+12 E_{1}\right)\left(p+D_{1}\right)^{3}}{72 p}-\frac{E_{1} p^{2}}{4}-\frac{D_{1}^{3}\left(p+4 D_{1}+12 E_{1}\right)}{72 p}
\end{array}\right], \\
& D_{2}=\frac{(1+p)(1-\alpha)}{200}, E_{2}=\frac{3(1+p)(\alpha-1)-100 p}{200}, G=\frac{\left(D_{2}-p\right)^{4}-\left(D_{2}+p\right)^{4}}{24 p} \text {, }
\end{aligned}
$$




$$
\begin{aligned}
& F=\frac{\left(D_{2}+p\right)^{4}+\left(D_{2}-p\right)^{4}}{24 p}, V=\frac{-\beta\left(p+D_{2}\right)^{6}+\beta\left(p-D_{2}\right)^{6}}{30 p^{3}}+12 \beta F-m^{2}\left(\frac{p}{180}+\frac{E_{2}}{6}-\frac{p+12 E_{2}}{12}\right), \\
& Z=\frac{\beta\left(p+D_{2}\right)^{6}-\beta\left(p-D_{2}\right)^{6}}{30 p^{2}}+\beta G+\frac{7 m^{2} D_{2}}{90}, \\
& W=\frac{-2 \beta\left(p+D_{2}\right)^{6}-2 \beta\left(p-D_{2}\right)^{6}}{3 p}+\frac{2 G\left(p+D_{2}\right)^{3}+2 G\left(p-D_{2}\right)^{3}}{3 p} \\
& -m^{2}\left[\begin{array}{l}
\frac{p^{3}}{9}+\frac{7 p D_{2}^{2}}{6}+2 E_{2} D_{2}^{2} \\
-\frac{D_{2}\left(p+D_{2}\right)^{3}+D_{2}\left(p-D_{2}\right)^{3}}{9 p}
\end{array}\right], \\
& W_{1}=\frac{2 \beta\left(p+D_{2}\right)^{6}-2 \beta\left(p-D_{2}\right)^{6}}{3 p}+\frac{2 G\left(p-D_{2}\right)^{3}-2 G\left(p+D_{2}\right)^{3}}{3 p} \\
& -m^{2}\left[\begin{array}{l}
\frac{2 p^{2} D_{2}}{3}+\frac{4 p^{2} E_{2}}{3}+2 E_{2} D_{2}^{2}+2 p E_{2} D_{2} \\
+\frac{D_{2}\left(p+D_{2}\right)^{3}-D_{2}\left(p-D_{2}\right)^{3}}{9 p}+\frac{2 D_{2}^{3}}{3}
\end{array}\right], \\
& D_{3}=\frac{(1+p)(\alpha-1)}{200}, E_{3}=\frac{3(1+p)(1-\alpha)-100 p}{200}, G_{1}=\frac{\left(D_{3}-p\right)^{4}-\left(D_{3}+p\right)^{4}}{24 p}, \\
& F_{1}=\frac{\left(D_{3}+p\right)^{4}+\left(D_{3}-p\right)^{4}}{24 p}, \\
& V_{1}=\frac{-\beta\left(p+D_{3}\right)^{6}+\beta\left(p-D_{3}\right)^{6}}{30 p^{3}}+12 \beta F_{1}-m^{2}\left(\frac{p}{180}+\frac{E_{3}}{6}-\frac{p+12 E_{3}}{12}\right), \\
& Z_{1}=\frac{\beta\left(p+D_{3}\right)^{6}-\beta\left(p-D_{3}\right)^{6}}{30 p^{2}}+\beta G_{1}+\frac{7 m^{2} D_{3}}{90}, \\
& W_{3}=\frac{-2 \beta\left(p+D_{3}\right)^{6}-2 \beta\left(p-D_{3}\right)^{6}}{3 p}+\frac{2 G_{1}\left(p+D_{3}\right)^{3}+2 G_{1}\left(p-D_{3}\right)^{3}}{3 p} \\
& -m^{2}\left[\begin{array}{l}
\frac{p^{3}}{9}+\frac{7 p D_{3}^{2}}{6}+2 E_{3} D_{3}^{2} \\
-\frac{D_{3}\left(p+D_{3}\right)^{3}+D_{3}\left(p-D_{3}\right)^{3}}{9 p}
\end{array}\right],
\end{aligned}
$$




$$
\begin{aligned}
& W_{2}= \frac{2 \beta\left(p+D_{3}\right)^{6}-2 \beta\left(p-D_{3}\right)^{6}}{3 p}+\frac{2 G_{1}\left(p-D_{3}\right)^{3}-2 G_{1}\left(p+D_{3}\right)^{3}}{3 p} \\
&-m^{2}\left[\begin{array}{l}
\frac{2 p^{2} D_{3}}{3}+\frac{4 p^{2} E_{3}}{3}+2 E_{3} D_{3}^{2}+2 p E_{3} D_{3} \\
+\frac{D_{3}\left(p+D_{3}\right)^{3}-D_{3}\left(p-D_{3}\right)^{3}}{9 p}+\frac{2 D_{3}^{3}}{3}
\end{array}\right] .
\end{aligned}
$$

